\title{
Classes of Bivariate Orthogonal Polynomials
}

\author{
Mourad E.H. ISMAIL ${ }^{\dagger}$ and Ruiming ZHANG $\S$ \\ $\dagger$ Department of Mathematics, King Saud University, Riyadh, Saudi Arabia \\ $\ddagger$ Department of Mathematics, University of Central Florida, Orlando, Florida 32816, USA \\ E-mail: mourad.eh.ismail@gmail.com \\ URL: http://math.cos.ucf.edu/people/ismail-mourad/ \\ $\S$ College of Science, Northwest A\&F University, Yangling, Shaanxi 712100, P.R. China \\ E-mail: ruimingzhang@yahoo.com
}

Received August 04, 2015, in final form February 15, 2016; Published online February 24, 2016

http://dx.doi.org/10.3842/SIGMA.2016.021

\begin{abstract}
We introduce a class of orthogonal polynomials in two variables which generalizes the disc polynomials and the $2-D$ Hermite polynomials. We identify certain interesting members of this class including a one variable generalization of the 2- $D$ Hermite polynomials and a two variable extension of the Zernike or disc polynomials. We also give $q$-analogues of all these extensions. In each case in addition to generating functions and three term recursions we provide raising and lowering operators and show that the polynomials are eigenfunctions of second-order partial differential or $q$-difference operators.
\end{abstract}

Key words: disc polynomials; Zernike polynomials; $2 D$-Laguerre polynomials; $q$ - $2 D$-Laguerre polynomials; generating functions; ladder operators; $q$-Sturm-Liouville equations; $q$-integrals; $q$-Zernike polynomials; $2 D$-Jacobi polynomials; $q$-2D-Jacobi polynomials; connection relations; biorthogonal functions; generating functions; Rodrigues formulas; zeros

2010 Mathematics Subject Classification: 33C50; 33D50; 33C45; 33D45

\section{Introduction}

One of the earliest orthogonal polynomials are the Legendre polynomials which are orthogonal with respect to $d x=$ (linear measure) on the unit interval. They are the spherical harmonics in $\mathbb{R}^{2}$. The ultraspherical polynomials are the spherical harmonics on $\mathbb{R}^{m}$ and are orthogonal with respect to $\frac{\Gamma(\nu+3 / 2)}{\sqrt{\pi} \Gamma(\nu+1)}\left(1-x^{2}\right)^{\nu} d x$ on the unit interval. If we replace $x$ by $x / \sqrt{\nu}$ and let $\nu \rightarrow \infty$ the measure becomes $\pi^{-1 / 2} e^{-x^{2}} d x$ and the ultraspherical polynomials, properly scaled, tend to Hermite polynomials. The next sequence of polynomials in this hierarchy are the Jacobi polynomials which are orthogonal on the unit interval with respect to $\frac{2^{-\alpha-\beta-1} \Gamma(\alpha+\beta+2)}{\Gamma(\alpha+1) \Gamma(\beta+1)}(1-$ $x)^{\alpha}(1+x)^{\beta} d x$. The Laguerre polynomials arise when $x$ is replaced by $-1+2 x / \alpha$ and $\alpha \rightarrow \infty$.

The $2 D$ history is somewhat parallel. In the late 1920's Frits Zernike was working on optical problems involving telescopes and microscopes. He introduced polynomials orthogonal on $|z| \leq 1$ with respect to the area measure, so this is the $2 D$ analogue of Legendre polynomials, see [38, 39]. The more general disc or Zernike polynomials are orthogonal with respect to $\left(1-x^{2}-y^{2}\right)^{\nu} d x d y$ on the unit disc. Again if we replace $(x, y)$ by $(x / \sqrt{\nu}, y / \sqrt{\nu})$ and let $\nu \rightarrow \infty$ this measure, properly normalized becomes $e^{-x^{2}-y^{2}} d x d y$ on $\mathbb{R}^{2}$. The polynomials become the $2 D$-Hermite polynomials introduced by Ito in [22] in a different way. They are defined by

$$
H_{m, n}\left(z_{1}, z_{2}\right)=\sum_{k=0}^{m \wedge n}\left(\begin{array}{l}
m \\
k
\end{array}\right)\left(\begin{array}{l}
n \\
k
\end{array}\right)(-1)^{k} k ! z^{m-k}(\bar{z})^{n-k},
$$

\footnotetext{
${ }^{\star}$ This paper is a contribution to the Special Issue on Orthogonal Polynomials, Special Functions and Applications. The full collection is available at http://www.emis.de/journals/SIGMA/OPSFA2015.html
} 
where $m \wedge n=\min \{m, n\}$. In 1966 D.R. Myrick [27] considered only the radial part of the polynomials orthogonal with respect to the Jacobi type measure $\left(x^{2}+y^{2}\right)^{\alpha}\left(1-x^{2}-y^{2}\right)^{\beta} d x d y$ on the unit disc, see also [36]. Addition theorem for the disc polynomials were found by Sapiro [29] and Koornwinder [24]. Maldonado [26] very briefly considered a class of radial functions which can be used to generate $2 D$ orthogonal polynomials. P. Floris [10] introduced a $q$-analogue of the disc polynomials proved an addition theorem for them, see also Floris and Koelink [11].

There are several other families of orthogonal polynomials in two and higher variables. Koornwinder's survey article [25] covers most of what was known up to the time of its publication in 1975. In the 19th century Hermite found families of orthogonal and biorthogonal polynomials, see $[14,15],[8$, Chapter 12] and [3, 4]. The more recent book [7] contains a treatment of the theory and applications of orthogonal polynomials in 2 and higher variables. One recent related reference is [33]. The $2 D$-Hermite polynomials have may applications to physical problems, see $[2,6,30,32,34,35]$. Mathematical properties of these polynomials have been developed in $[12,13,16]$. A multilinear generating function, of Kibble-Slepian type, is proved in [18]. In [21] we gave two $q$-analogues of the $2 D$-Hermite polynomials and studied the polynomials in great detail. We also introduced a different $q$-analogue of the disc polynomials.

In this paper we first identify a general class of two variable polynomials whose measure is the product of the uniform measure on the circle times a radial measure. This class not only include the $2 D$-Hermite polynomials and their $q$-analogues but it also contains the Zernike (or disc) polynomials and its $q$-analogues. This will be done in Section 2. One special feature of this class is that multiplication by $z$ or $\bar{z}$ results in a three term recurrence relation. This special feature is unlike what happens for general real $2-D$ systems [7]. In fact the earlier work [18] motivated Yuan $\mathrm{Xu}$ [37] to reformulate the theory of orthogonal polynomials in several variables in terms of complex variables.

In Section 3 we give a one parameter extension of the $2 D$-Hermite polynomials. They are $2 D$ analogues of Laguerre polynomials and will be denoted by $\left\{Z_{m, n}^{(\beta)}(z, \bar{z})\right\}$. We record their definition, orthogonality relation, and the three term recurrence relations in Section 3. In Section 3 we also derive several differential properties of these polynomials. It must be noted that the combinatorics of these polynomials have been explored in [19]. Section 4 contains a treatment of a two parameter family of disc polynomials. They are orthogonal with respect to $\left(x^{2}+y^{2}\right)^{\alpha}\left(1-x^{2}-y^{2}\right)^{\beta} d x d y$ on the unit disc. They were briefly studied in [20] but here we derive new generating functions and study their differential-difference properties and show that they satisfy a Sturm-Liouville system.

The $q$-analogues start with Section 5 where we introduce two $q$-analogues of the $2 D$-Laguerre polynomials $\left\{Z_{m, n}^{(\beta)}(z, \bar{z} \mid q)\right\}$. Both can be considered as a $q$-extension of the $2 D$-Laguerre polynomials, one for $q \in(0,1)$ and one for $q>1$. As expected the case $q>1$ leads to an indeterminate moment problem while the case $q \in(0,1)$ is a $2 D$-extension of the Wall or little $q$-Laguerre polynomials, $[5,23]$. A $q$-analogue of the two parameter $2 D$-disc polynomials is introduced in Section 6 and many of their properties are developed. In Section 7 we find polynomial solutions of a second-order partial differential equation. Section 8 contains a system of biorthogonal polynomials which is a possible $2 D$-analogue of the Askey-Wilson polynomials.

We shall use the notations

$$
\begin{aligned}
& \left(D_{q, z} f\right)(z)=\frac{f(z)-f(q z)}{(1-q) z}, \quad\left(\delta_{q, z} f\right)(z)=z\left(D_{q, z} f\right)(z), \\
& {[\alpha]_{q}=\frac{1-q^{\alpha}}{1-q}, \quad m \vee n=\max \{m, n\}, \quad m \wedge n=\min \{m, n\}}
\end{aligned}
$$

and

$$
\partial_{z}=\frac{\partial}{\partial_{z}}, \quad \delta_{z}=z \partial_{z}
$$


Throughout the paper we will use $z_{1}$ and $z_{2}$. The polynomials we consider here are polynomials of two real variables, so we shall always assume that $z_{2}=\bar{z}_{1}$.

\section{General constructions}

We now give a concrete construction for general $2 D$ systems. Given $N \in \mathbb{N} \cup\{\infty\}$, let $\nu(\theta ; N)$ be a probability measure on the circle $\mathbb{T}=\left\{e^{i \theta} \mid 0 \leq \theta \leq 2 \pi\right\}$ of Fourier type

$$
\int_{\mathbb{T}} e^{i(m-n) \theta} d \nu(\theta ; N)=\delta_{m, n}, \quad m, n=0,1, \ldots, N
$$

and $\left\{\phi_{n}(r ; \alpha)\right\}$ be a system of orthogonal polynomials satisfying the orthogonality relation

$$
\int_{0}^{\infty} \phi_{m}(r ; \alpha) \phi_{n}(r ; \alpha) r^{\alpha} d \mu(r)=\zeta_{n}(\alpha) \delta_{m, n}, \quad \alpha \geq 0 .
$$

It is assumed the positive Borel measure $\mu$ does not depend on $\alpha$. Let

$$
\phi_{n}(r ; \alpha)=\sum_{j=0}^{n} c_{j}(n, \alpha) r^{n-j}, \quad c_{j}(n, \alpha) \in \mathbb{R},
$$

and define polynomials

$$
f_{m, n}\left(z_{1}, z_{2} ; \beta\right)= \begin{cases}z_{1}^{m-n} \phi_{n}\left(z_{1} z_{2} ; m-n+\beta\right), & m \geq n, \\ f_{n, m}\left(z_{2}, z_{1} ; \beta\right), & m<n .\end{cases}
$$

This defines the polynomials for all $m, n$ and $z_{1}, z_{2} \in \mathbb{C}$. It is clear that

$$
\overline{f_{m, n}(z, \bar{z} ; \beta)}=f_{n, m}(z, \bar{z} ; \beta), \quad m<n .
$$

From (2.2) it is clear that for $m \geq n$

$$
z_{1} f_{m, n}\left(z_{1}, z_{2} ; \beta+1\right)=f_{m+1, n}\left(z_{1}, z_{2} ; \beta\right),
$$

and

$$
-i \frac{\partial}{\partial \theta} f_{m, n}(z, \bar{z} ; \beta)=(m-n) f_{m, n}(z, \bar{z} ; \beta)
$$

Therefore,

$$
\left(\delta_{z_{1}}-\delta_{z_{2}}\right) f_{m, n}\left(z_{1}, z_{2} ; \beta\right)=(m-n) f_{m, n}\left(z_{1}, z_{2} ; \beta\right), \quad m \geq n .
$$

Similarly,

$$
\left(\delta_{q, z_{1}}-q^{m-n} \delta_{q, z_{2}}\right) f_{m, n}\left(z_{1}, z_{2} ; \beta\right)=[m-n]_{q} f_{m, n}\left(z_{1}, z_{2} ; \beta\right), \quad m \geq n .
$$

It must be noted that $f_{m, n}\left(z_{1}, z_{2}\right)$ is defined for $z_{1}, z_{2} \in \mathbb{C}$ but the next theorem gives an orthogonality relation on $\mathbb{R}^{2}$. This is exactly analogous to the classical univariate orthogonal polynomials which are defined for $z \in \mathbb{C}$ but their orthogonality is on a subset of $\mathbb{R}$.

Theorem 2.1. Given $N \in \mathbb{N}$, for nonnegative integers $m, n, s, t$ such that $m+t \leq N, n+s \leq N$ the polynomials $\left\{f_{m, n}\left(z_{1}, z_{2} ; \beta\right)\right\}$ satisfy the orthogonality relation

$$
\int_{\mathbb{R}^{2}} f_{m, n}(z, \bar{z} ; \beta) \overline{f_{s, t}(z, \bar{z} ; \beta)} d \nu(\theta ; N) d \mu\left(r^{2} ; \beta\right)=\zeta_{m \wedge n}(|m-n|+\beta) \delta_{m, s} \delta_{n, t},
$$

where $d \mu(r ; \beta)=r^{\beta} d \mu(r)$. If $N=\infty$, (2.4) holds for all nonnegative integers $m, n, s, t$. 
Proof. There is no loss of generality in assuming $m \geq n$. Otherwise, we simply apply (2.3) to switch the orders of $m$ and $n, s$ and $t$, then consider the consider the complex conjugate of the obtained integral instead. By performing the integral over $\theta$ we see that the left-hand side of (2.4) equals

$$
\int_{\mathbb{R}^{2}} f_{m, n}(z, \bar{z} ; \beta) \overline{f_{s, t}(z, \bar{z} ; \beta)} d \nu(\theta) d \mu\left(r^{2} ; \beta\right)=0
$$

unless $m+t=n+s$. Let $\alpha=m-n=s-t$. Therefore the above integral equals

$$
\begin{aligned}
& \int_{0}^{\infty} \sum_{j=0}^{n} c_{j}(n, \alpha+\beta) r^{2 n+\alpha-2 j} \sum_{k=0}^{t} c_{k}(t, \alpha+\beta) r^{2 t+\alpha-2 k} r^{2 \beta} d \mu\left(r^{2}\right) \\
& \quad=\int_{0}^{\infty} \sum_{j=0}^{n} c_{j}(n, \alpha+\beta) r^{n-j} \sum_{k=0}^{t} c_{k}(t, \alpha+\beta) r^{t-k} r^{\alpha+\beta} d \mu(r)=\zeta_{n}(\alpha+\beta) \delta_{n, t},
\end{aligned}
$$

and the proof is complete.

Remark 2.2. For any positive integer $N$, if we take $\nu(\theta ; N)$ as the discrete probability measure used in FFT (fast fourier transform), we get finitely many $2 \mathrm{D}$ orthogonal polynomials. But in most of our applications we use $N=\infty$ and the circle measure $\frac{d \theta}{2 \pi}$ for $d \nu(\theta)$ unless specifically stated.

We now come to the three term recurrence relation.

Theorem 2.3. The polynomials $\left\{f_{m, n}\left(z_{1}, z_{2} ; \beta\right)\right\}$ satisfy the three term recurrence relation

$$
\begin{aligned}
& z_{2} f_{m+1, n}\left(z_{1}, z_{2} ; \beta\right)=\frac{c_{0}(n, m-n+1+\beta)}{c_{0}(n+1, m-n+\beta)} f_{m+1, n+1}\left(z_{1}, z_{2} ; \beta\right) \\
& \quad-\frac{c_{0}(n, m-n+1+\beta) c_{n+1}(n+1, m-n+\beta)}{c_{0}(n+1, m-n+\beta) c_{n}(n, m-n+\beta)} f_{m, n}\left(z_{1}, z_{2} ; \beta\right), \quad m \geq n .
\end{aligned}
$$

Proof. Since the polynomials $\left\{\phi_{n}(r ; \alpha)\right\}$ are orthogonal on $[0, \infty)$, their zeros are in $(0, \infty)$. Hence $\phi_{n}(0 ; \alpha) \neq 0$ for all $n$ and all $\alpha \geq 0$. From the Christoffel formula [17, Theorem 2.7.1] we see that

$$
r \phi_{n}(r ; \alpha+1)=\frac{c_{0}(n, \alpha+1)}{c_{0}(n+1, \alpha) \phi_{n}(0 ; \alpha)}\left[\phi_{n+1}(r ; \alpha) \phi_{n}(0 ; \alpha)-\phi_{n}(r ; \alpha) \phi_{n+1}(0 ; \alpha)\right],
$$

that is

$$
r \phi_{n}(r ; \alpha+1)=\frac{c_{0}(n, \alpha+1)}{c_{0}(n+1, \alpha)} \phi_{n+1}(r ; \alpha)-\frac{c_{0}(n, \alpha+1) c_{n+1}(n+1, \alpha)}{c_{0}(n+1, \alpha) c_{n}(n, \alpha)} \phi_{n}(r ; \alpha) .
$$

Now take $\alpha=m-n+\beta$, replace $r$ by $z_{1} z_{2}$ then multiply both sides by $z_{1}{ }^{m-n}$ to establish (2.5).

One can also prove that $[20]$

$$
z_{1} f_{m, n}\left(z_{1}, z_{2} ; \beta\right)-\frac{c_{0}(n, m-n+\beta)}{c_{0}(n, m+1-n+\beta)} f_{m+1, n}\left(z_{1}, z_{2} ; \beta\right)=u_{m, n} f_{m, n-1}\left(z_{1}, z_{2} ; \beta\right)
$$

where

$$
u_{m, n}=\frac{c_{0}(n, \alpha+1) c_{1}(n, \alpha)-c_{0}(n, \alpha) c_{1}(n, 1+\alpha)}{c_{0}(n-1,1+\alpha) c_{0}(n, 1+\alpha)}
$$

and $\alpha=m-n+\beta$. 
It must be noted that the $2 D$-polynomials may have recurrence relations containing more than three terms [7]. It is surprising that the polynomials in the special class we are considering have three term recursions. Another important point is that the definition of the polynomials $f_{m, n}$ indicates that it has either a trivial zero at $z=0$ or the zeros will lie on circles whose radii are the square roots of the zeros of $\phi_{n}(r ; m-n+\beta)$. The zeros of $\phi_{n}(r ; m-n+\beta)$ are positive, real and simple.

Let

$$
r \phi_{n}(r ; \alpha)=a_{n}(\alpha) \phi_{n+1}(r ; \alpha)+c_{n}(\alpha) \phi_{n}(r ; \alpha)+b_{n}(\alpha) \phi_{n-1}(r ; \alpha)
$$

be the three term recurrence relation satisfied by the polynomials $\left\{\phi_{n}(r ; \alpha)\right\}$, for some real numbers $a_{n}(\alpha), b_{n}(\alpha), c_{n}(\alpha)$. By matching the corresponding coefficients we find that

$$
\begin{aligned}
& a_{n}(\alpha)=\frac{c_{0}(n, \alpha)}{c_{0}(n+1, \alpha)} \\
& b_{n}(\alpha)=\frac{c_{0}(n, \alpha) c_{2}(n, \alpha)-c_{1}(n, \alpha)^{2}}{c_{0}(n-1, \alpha) c_{0}(n, \alpha)}-\frac{c_{0}(n, \alpha) c_{2}(n+1, \alpha)-c_{1}(n, \alpha) c_{1}(n+1, \alpha)}{c_{0}(n-1, \alpha) c_{0}(n+1, \alpha)}, \\
& c_{n}(\alpha)=\frac{c_{1}(n, \alpha)}{c_{0}(n, \alpha)}-\frac{c_{1}(n+1, \alpha)}{c_{0}(n+1, \alpha)} .
\end{aligned}
$$

Making use of (2.1) and (2.2) we conclude that the recursion (2.8) becomes

$$
\begin{aligned}
{\left[z_{1} z_{2}\right.} & \left.-c_{n}(m-n+\beta)\right] f_{m, n}\left(z_{1}, z_{2} ; \beta\right) \\
& =a_{n}(m-n+\beta) f_{m+1, n+1}\left(z_{1}, z_{2} ; \beta\right)+b_{n}(m-n+\beta) f_{m-1, n-1}\left(z_{1}, z_{2} ; \beta\right) .
\end{aligned}
$$

Example 2.4. Consider the case of the 2- $D$ Hermite polynomials when the coefficients in (2.2) are given by

$$
c_{j}(n, \alpha)=\frac{n !(n+\alpha) !(-1)^{j}}{j !(n-j) !(n+\alpha-j) !} .
$$

The recursion (2.5) leads to the second three term recurrence relation

$$
\begin{aligned}
& z_{1} H_{m, n}\left(z_{1}, z_{2}\right)=n H_{m, n-1}\left(z_{1}, z_{2}\right)+H_{m+1, n}\left(z_{1}, z_{2}\right), \\
& z_{2} H_{m, n}\left(z_{1}, z_{2} \mid q\right)=m H_{m-1, n}\left(z_{1}, z_{2}\right)+H_{m, n+1}\left(z_{1}, z_{2}\right),
\end{aligned}
$$

while the first can be proved by direct computation.

Similarly the case when the coefficients in (2.2) are given by

$$
c_{j}(n, \alpha)=\frac{(q ; q)_{n}(q ; q)_{n+\alpha}(-1)^{j}}{(q ; q)_{j}(q ; q)_{n-j}(q ; q)_{n+\alpha-j}} q^{\left(\frac{j}{2}\right)}
$$

leads to the recurrence relations for this $q$-analogue, see [21].

Remark 2.5. If we demand that both (2.2) and (2.3) are valid for all $m, n, z$, then we must have

$$
c_{j}(n, \alpha)=\left(\begin{array}{c}
n \\
j
\end{array}\right) \frac{a_{j}(n, \alpha)}{(n+\alpha-j) !}
$$

for the classical cases, and

$$
c_{j}(n, \alpha)=\left(\begin{array}{l}
n \\
j
\end{array}\right) \frac{a_{j}(n, \alpha \mid q)}{(q ; q)_{n+\alpha-j}}
$$

for the $q$-analogues, where $c_{j}(n, \alpha)$ are the coefficients in (2.2). These factorizations imply that $\phi_{n}(r ; \alpha)$ must be a generalization of Laguerre orthogonal polynomials. However, in most of the cases, (2.2) is only valid for $m \geq n$, whereas (2.3) acts as a kind of analytical continuation to the case $m<n$. 
Remark 2.6. In view of the defining equation (2.2) the question of finding the large $m, n$ asymptotics of the two variate polynomials $f_{m, n}$ is equivalent to finding the large $m, n$ behavior of $\phi_{n}(r, m-n+\beta)$. It will be of interest to carry out this program at least for some special systems, including the Freud type polynomials orthogonal with respect to $x^{\alpha} \exp (-p(x))$, where $p$ is a polynomial with positive leading term.

We now consider an inverse to (2.6).

Proposition 2.7. Given a nonnegative measure on $(0, \infty)$

$$
d \mu(x ; \alpha)=x^{\alpha} d \nu(x), \quad x \geq 0, \alpha>-1
$$

such that

$$
\int_{0}^{\infty} x^{n} d \mu(x ; \alpha)<\infty, \quad n=0,1, \ldots
$$

Let $\left\{\phi_{n}(x ; \alpha)\right\}_{n=0}^{\infty}$ satisfy

$$
\int_{0}^{\infty} \phi_{m}(x ; \alpha) \phi_{n}(x ; \alpha) d \mu(x ; \alpha)=\zeta_{n}(\alpha) \delta_{m, n} .
$$

Then

$$
\phi_{n}(x ; \alpha)-a_{n}(\alpha) \phi_{n}(x ; \alpha+1)=b_{n}(\alpha) \phi_{n-1}(x ; \alpha+1),
$$

where

$$
\begin{aligned}
& \phi_{n}(x ; \alpha)=\sum_{j=0}^{n} c_{j}(n, \alpha) x^{n-j}, \\
& b_{n}(\alpha)=\frac{c_{0}(n, \alpha+1) c_{1}(n, \alpha)-c_{0}(n, \alpha) c_{1}(n, \alpha+1)}{c_{0}(n-1, \alpha+1) c_{0}(n, \alpha+1)}, \\
& a_{n}(\alpha)=\frac{c_{0}(n, \alpha)}{c_{0}(n, \alpha+1)} .
\end{aligned}
$$

Furthermore,

$$
\int_{0}^{\infty} x \phi_{n}(x ; \alpha) \phi_{n-1}(x ; \alpha+1) d \mu(x ; \alpha)=b_{n}(\alpha) \zeta_{n-1}(\alpha+1) .
$$

Proof. It is clear that $\phi_{n}(x ; \alpha)-a_{n}(\alpha) \phi_{n}(x ; \alpha+1)$ is a polynomial of degree at most $n-1$, then

$$
\phi_{n}(x ; \alpha)-a_{n}(\alpha) \phi_{n}(x ; \alpha+1)=\sum_{j=0}^{n-1} \alpha_{j} \phi_{j}(x ; \alpha+1),
$$

where

$$
\begin{aligned}
\alpha_{j} \zeta_{j}(\alpha+1) & =\int_{0}^{\infty}\left\{\phi_{n}(x ; \alpha)-a_{n}(\alpha) \phi_{n}(x ; \alpha+1)\right\} \phi_{j}(x ; \alpha+1) d \mu(x ; \alpha+1) \\
& =\int_{0}^{\infty} \phi_{n}(x ; \alpha) \phi_{j}(x ; \alpha+1) d \mu(x ; \alpha+1) \\
& =\int_{0}^{\infty} x \phi_{n}(x ; \alpha) \phi_{j}(x ; \alpha+1) d \mu(x ; \alpha) .
\end{aligned}
$$

Clearly $\alpha_{j}=0$ for $0 \leq j \leq n-2$ and $\alpha_{n-1}=b_{n}(\alpha)$. Then $b_{n}(\alpha)$ is obtained by matching the leading coefficients of two sides. 
Corollary 2.8. Under the assumptions of Proposition 2.7, let

$$
\phi_{n}(x ; \alpha+1)=\sum_{j=0}^{n} \lambda_{j}(n, \alpha) \phi_{j}(x ; \alpha) .
$$

Then

$$
\begin{aligned}
& \lambda_{j}(n, \alpha)=\frac{(-1)^{n-j}}{a_{j}(\alpha)} \prod_{k=0}^{n-j-1} \frac{b_{n-k}(\alpha)}{a_{n-k}(\alpha)}, \quad 0 \leq j \leq n-1, \\
& \lambda_{n}(n, \alpha)=\frac{1}{a_{n}(\alpha)}
\end{aligned}
$$

and

$$
\frac{\zeta_{n}(\alpha)}{\zeta_{0}(\alpha+n)}=\prod_{j=0}^{n-1} \frac{b_{n-j}(\alpha+j) c_{0}(n-j, \alpha+j)}{a_{n-j-1}(\alpha+j) c_{0}(n-j-1, \alpha+j)} .
$$

Furthermore, we have

$$
d_{n}(\alpha) \phi_{n}(x ; \alpha)=\phi_{n}(x ; \alpha+1)-x \sum_{j=0}^{n-1} f_{j}(n, \alpha) \phi_{j}(x ; \alpha+1)
$$

and

$$
\phi_{n}(x ; \alpha)=\phi_{n}(0 ; \alpha)-x \sum_{j=0}^{n-1} g_{j}(n, \alpha) \phi_{j}(x ; \alpha+1),
$$

where

$$
\begin{aligned}
& d_{n}(\alpha)=\frac{c_{n}(n, \alpha+1)}{c_{n}(n, \alpha)} \\
& f_{k}(n, \alpha)=\sum_{j=0}^{k} \frac{\lambda_{j}(n, \alpha) \lambda_{j}(k, \alpha) \zeta_{j}(\alpha+1)}{\zeta_{k}(\alpha+1)}, \quad 0 \leq k \leq n-1, \\
& g_{k}(n, \alpha)=\frac{(-1)^{k} \zeta_{0}(\alpha) c_{0}(0, \alpha+1) c_{n}(n, \alpha)}{\zeta_{k}(\alpha+1)} \prod_{j=0}^{k-1} \frac{b_{k-j}(\alpha)}{a_{k-j}(\alpha)} .
\end{aligned}
$$

Proof. From (2.16) and (2.14) we get

$$
\phi_{n}(x ; \alpha)-a_{n}(\alpha) \sum_{j=0}^{n} \lambda_{j}(n, \alpha) \phi_{j}(x ; \alpha)=b_{n}(\alpha) \sum_{j=0}^{n-1} \lambda_{j}(n-1, \alpha) \phi_{j}(x ; \alpha) .
$$

Then

$$
1-\lambda_{n}(n, \alpha) a_{n}(\alpha)=0
$$

and

$$
-a_{n}(\alpha) \lambda_{j}(n, \alpha)=b_{n}(\alpha) \lambda_{j}(n-1, \alpha)
$$

for $0 \leq j \leq n-1$, which lead to (2.18) and (2.17) respectively. 
From (2.15) we get (2.16) we get

$$
\begin{aligned}
b_{n}(\alpha) \zeta_{n-1}(\alpha+1) & =\lambda_{n-1}(n-1, \alpha) \int_{0}^{\infty} x \phi_{n}(x ; \alpha) \phi_{n-1}(x ; \alpha) d \mu(x ; \alpha) \\
& =\frac{1}{a_{n-1}(\alpha)} \int_{0}^{\infty} x \phi_{n}(x ; \alpha) \phi_{n-1}(x ; \alpha) d \mu(x ; \alpha) .
\end{aligned}
$$

Since $\left\{\phi_{n}(x ; \alpha)\right\}_{n=0}^{\infty}$ are orthogonal polynomials with respective to the measure $d \mu(x ; \alpha)$, hence

$$
x \phi_{n-1}(x ; \alpha)=\eta_{n} \phi_{n}(x ; \alpha)+\delta_{n} \phi_{n-1}(x ; \alpha)+\epsilon_{n} \phi_{n-2}(x ; \alpha),
$$

where

$$
\eta_{n}=\frac{c_{0}(n-1, \alpha)}{c_{0}(n, \alpha)}
$$

Then

$$
b_{n}(\alpha) \zeta_{n-1}(\alpha+1)=\frac{\eta_{n} \zeta_{n}(\alpha)}{a_{n-1}(\alpha)},
$$

and

$$
\begin{aligned}
\zeta_{n}(\alpha) & =\frac{b_{n}(\alpha)}{a_{n-1}(\alpha)} \frac{c_{0}(n, \alpha)}{c_{0}(n-1, \alpha)} \zeta_{n-1}(\alpha+1) \\
& =\zeta_{0}(\alpha+n) \prod_{j=0}^{n-1} \frac{b_{n-j}(\alpha+j)}{a_{n-j-1}(\alpha+j)} \frac{c_{0}(n-j, \alpha+j)}{c_{0}(n-j-1, \alpha+j)},
\end{aligned}
$$

which gives (2.19).

Let

$$
\phi_{n}(x ; \alpha+1)-d_{n}(\alpha) \phi_{n}(x ; \alpha)=x \sum_{j=0}^{n-1} f_{j}(n, \alpha) \phi_{j}(x ; \alpha+1) .
$$

For $0 \leq k \leq n-1$ we have

$$
\begin{aligned}
\int_{0}^{\infty} & \left\{\phi_{n}(x, \alpha+1)-d_{n}(\alpha) \phi_{n}(x, \alpha)\right\} \phi_{k}(x, \alpha+1) d \mu(x ; \alpha) \\
& =\int_{0}^{\infty}\left\{x \sum_{j=0}^{n-1} f_{j}(n, \alpha) \phi_{j}(x, \alpha+1)\right\} \phi_{k}(x, \alpha+1) d \mu(x ; \alpha) \\
& =\int_{0}^{\infty}\left\{\sum_{j=0}^{n-1} f_{j}(n, \alpha) \phi_{j}(x, \alpha+1)\right\} \phi_{k}(x, \alpha+1) d \mu(x ; \alpha+1) \\
& =f_{k}(n, \alpha) \zeta_{k}(\alpha+1),
\end{aligned}
$$

which is

$$
\begin{aligned}
f_{k}(n, \alpha) \zeta_{k}(\alpha+1) & =\int_{0}^{\infty} \phi_{n}(x, \alpha+1) \phi_{k}(x, \alpha+1) d \mu(x ; \alpha) \\
& =\sum_{j=0}^{k} \lambda_{j}(n, \alpha) \lambda_{j}(k, \alpha) \zeta_{j}(\alpha+1)
\end{aligned}
$$


or

$$
f_{k}(n, \alpha)=\sum_{j=0}^{k} \frac{\lambda_{j}(n, \alpha) \lambda_{j}(k, \alpha) \zeta_{j}(\alpha+1)}{\zeta_{k}(\alpha+1)} .
$$

On the other hand, let

$$
\phi_{n}(x ; \alpha)=\phi_{n}(0 ; \alpha)-x \sum_{j=0}^{n-1} g_{j}(n, \alpha) \phi_{j}(x ; \alpha+1),
$$

then, for $0 \leq k \leq n-1$, we have

$$
\begin{aligned}
\int_{0}^{\infty} & \left\{\phi_{n}(x ; \alpha)-\phi_{n}(0 ; \alpha)\right\} \phi_{k}(x, \alpha+1) d \mu(x ; \alpha) \\
& =-\int_{0}^{\infty}\left\{x \sum_{j=0}^{n-1} g_{j}(n, \alpha) \phi_{j}(x ; \alpha+1)\right\} \phi_{k}(x, \alpha+1) d \mu(x ; \alpha) \\
& =-\int_{0}^{\infty}\left\{\sum_{j=0}^{n-1} g_{j}(n, \alpha) \phi_{j}(x ; \alpha+1)\right\} \phi_{k}(x, \alpha+1) d \mu(x ; \alpha+1) \\
& =-g_{k}(n, \alpha) \zeta_{k}(\alpha+1),
\end{aligned}
$$

that is

$$
\begin{aligned}
g_{k}(n, \alpha) \zeta_{k}(\alpha+1) & =\int_{0}^{\infty} \phi_{n}(0 ; \alpha) \phi_{k}(x, \alpha+1) d \mu(x ; \alpha) \\
& =\phi_{n}(0 ; \alpha) \lambda_{0}(k, \alpha) \phi_{0}(0, \alpha) \zeta_{0}(\alpha)=c_{n}(n, \alpha) c_{0}(0, \alpha) \zeta_{0}(\alpha) \lambda_{0}(k, \alpha) \\
& =(-1)^{k} \zeta_{0}(\alpha) c_{0}(0, \alpha+1) c_{n}(n, \alpha) \prod_{j=0}^{k-1} \frac{b_{k-j}(\alpha)}{a_{k-j}(\alpha)}
\end{aligned}
$$

that is

$$
g_{k}(n, \alpha)=\frac{(-1)^{k} \zeta_{0}(\alpha) c_{0}(0, \alpha+1) c_{n}(n, \alpha)}{\zeta_{k}(\alpha+1)} \prod_{j=0}^{k-1} \frac{b_{k-j}(\alpha)}{a_{k-j}(\alpha)} .
$$

Corollary 2.9. Let $\phi_{n}(x, \alpha), a_{n}(\alpha), b_{n}(\alpha)$ as in Proposition 2.7 , for $m \geq n-1$ the equation (2.7) is in the form

$$
z_{1} f_{m, n}\left(z_{1}, z_{2}\right)-v_{m, n} f_{m+1, n}\left(z_{1}, z_{2}\right)=u_{m, n} f_{m, n-1}\left(z_{1}, z_{2}\right)
$$

where

$$
u_{m, n}=b_{n}(m-n), \quad v_{m, n}=a_{n}(m-n) .
$$

Proof. For $m \geq n-1$, applying the definition $f_{m, n}\left(z_{1}, z_{2}\right)=z_{1}^{m-n} \phi_{n}\left(z_{1} z_{2} ; m-n\right), m \geq n$ to get (2.20).

We next consider differential or $q$-difference equations satisfied by $f_{m, n}\left(z_{1}, z_{2}\right)$. 
Theorem 2.10. Assume that $\phi_{n}(r ; \alpha)$ satisfies the second-order differential equation

$$
A_{n}(r, \alpha) \delta_{r}^{2} f+B_{n}(r, \alpha) \delta_{r} f+C_{n}(r, \alpha) f=0,
$$

where

$$
\delta_{r} f=r \frac{\partial}{\partial r} f
$$

Then $f_{m, n}\left(z_{1}, z_{2} ; \beta\right)$, for $\alpha=m-n+\beta$ and $m \geq n$, satisfies the second-order partial differential equations

$$
\begin{aligned}
& A_{n}\left(z_{1} z_{2}, \alpha\right) \delta_{z_{2}}^{2} f+B_{n}\left(z_{1} z_{2}, \alpha\right) \delta_{z_{2}} f+C_{n}\left(z_{1} z_{2}, \alpha\right) f=0, \\
& A_{n}\left(z_{1} z_{2}, \alpha\right) \delta_{z_{1}}^{2} f+\left\{B_{n}\left(z_{1} z_{2}, \alpha\right)-2 \alpha A_{n}\left(z_{1} z_{2}, \alpha\right)\right\} \delta_{z_{1}} f \\
& \quad+\left\{\alpha^{2} A_{n}\left(z_{1} z_{2}, \alpha\right)-\alpha B_{n}\left(z_{1} z_{2}, \alpha\right)+C_{n}\left(z_{1} z_{2}, \alpha\right)\right\} f=0 .
\end{aligned}
$$

Similarly, assume that $\phi_{n}(r ; \alpha)$ satisfies the second order $q$-difference equation

$$
A_{n}(r, \alpha) \delta_{q, r}^{2} f+B_{n}(r, \alpha) \delta_{q, r} f+C_{n}(r, \alpha) f=0,
$$

where

$$
\delta_{q, r} f=r D_{q, r} f .
$$

Then $f_{m, n}\left(z_{1}, z_{2}\right)$, for $m \geq n$ and $\alpha=m-n+\beta$, satisfies the q-partial difference equations

$$
\begin{aligned}
& A_{n}\left(z_{1} z_{2}, \alpha\right) \delta_{q, z_{2}}^{2} f+B_{n}\left(z_{1} z_{2}, \alpha\right) \delta_{q, z_{2}} f+C_{n}\left(z_{1} z_{2}, \alpha\right) f=0, \\
& A_{n}\left(z_{1} z_{2}, \alpha\right) \delta_{q, z_{1}}^{2} f+\left\{q^{\alpha} B_{n}\left(z_{1} z_{2}, \alpha\right)-2[\alpha]_{q} A_{n}\left(z_{1} z_{2}, \alpha\right)\right\} \delta_{q, z_{1}} f \\
& \quad+\left\{[\alpha]_{q}^{2} A_{n}\left(z_{1} z_{2}, \alpha\right)-[\alpha]_{q} q^{\alpha} B_{n}\left(z_{1} z_{2}, \alpha\right)+q^{2 \alpha} C_{n}\left(z_{1} z_{2}, \alpha\right)\right\} f=0 .
\end{aligned}
$$

Proof. Observe that

$$
\begin{aligned}
& \phi_{n}\left(z_{1} z_{2}\right)=z_{1}^{-\alpha} f_{m, n}\left(z_{1}, z_{2}\right), \\
& \delta_{z_{1} z_{2}} \phi_{n}\left(z_{1} z_{2}\right)=z_{1}^{-\alpha} \delta_{z_{2}} f_{m, n}\left(z_{1}, z_{2}\right)=z_{1}^{-\alpha}\left\{\delta_{z_{1}}-\alpha\right\} f \\
& \delta_{z_{1} z_{2}}^{2} \phi_{n}\left(z_{1} z_{2}\right)=z_{1}^{-\alpha} \delta_{z_{2}}^{2} f_{m, n}\left(z_{1}, z_{2}\right)=z_{1}^{-\alpha}\left\{\delta_{z_{1}}^{2}-2 \alpha \delta_{z_{1}}+\alpha^{2}\right\} f \\
& \delta_{q, z_{1} z_{2}} \phi_{n}\left(z_{1} z_{2}\right)=z_{1}^{-\alpha} \delta_{q, z_{2}} f_{m, n}\left(z_{1}, z_{2}\right)=q^{-\alpha} z_{1}^{-\alpha}\left\{\delta_{q, z_{1}}-[\alpha]_{q}\right\} f \\
& \delta_{q, z_{1} z_{2}}^{2} \phi_{n}\left(z_{1} z_{2}\right)=z_{1}^{-\alpha} \delta_{q, z_{2}}^{2} f_{m, n}\left(z_{1}, z_{2}\right)=q^{-2 \alpha} z_{1}^{-\alpha}\left\{\delta_{q, z_{1}}^{2}-2[\alpha]_{q} \delta_{q, z_{1}}+[\alpha]_{q}^{2}\right\} f .
\end{aligned}
$$

Then the proof of theorem follows from substituting the required combinations into the corresponding differential equation or $q$-difference equation for $\phi(r, \alpha)$.

A simple application of Fubini's theorem establishes the following theorem concerning generating functions.

Theorem 2.11. For $z_{1}, z_{2}, u, v \in \mathbb{C}$ such that

$$
\sum_{j=0}^{\infty} \frac{\left|u z_{1}\right|^{j}}{j !}\left|g_{j}\left(z_{1} z_{2}, u v\right)\right|<\infty, \quad \sum_{j=0}^{\infty} \frac{\left|u z_{1}\right|^{j}}{(q ; q)_{j}}\left|g_{j}\left(z_{1} z_{2}, u v\right)\right|<\infty,
$$

where

$$
g_{\alpha}(x, z)=\sum_{k=0}^{\infty} \varphi_{k}(x ; \alpha) z^{k}
$$


Then

$$
\begin{aligned}
& \sum_{m \geq n \geq 0} \frac{u^{m} v^{n}}{(m-n) !} f_{m, n}\left(z_{1}, z_{2}\right)=\sum_{j=0}^{\infty} \frac{\left(u z_{1}\right)^{j}}{j !} g_{j}\left(z_{1} z_{2}, u v\right), \\
& \sum_{m \geq n \geq 0} \frac{u^{m} v^{n}}{(q ; q)_{m-n}} f_{m, n}\left(z_{1}, z_{2} \mid q\right)=\sum_{j=0}^{\infty} \frac{\left(u z_{1}\right)^{j}}{(q ; q)_{j}} g_{j}\left(z_{1} z_{2}, u v\right) .
\end{aligned}
$$

Similarly, for $z_{1}, z_{2}, u, v \in \mathbb{C}$ such that

$$
\sum_{j=0}^{\infty}\left|u z_{1}\right|^{j}\left|g_{j}\left(z_{1} z_{2}, u v\right)\right|<\infty
$$

then

$$
\begin{aligned}
& \sum_{m \geq n \geq 0} u^{m} v^{n} f_{m, n}\left(z_{1}, z_{2}\right)=\sum_{j=0}^{\infty}\left(u z_{1}\right)^{j} g_{j}\left(z_{1} z_{2}, u v\right), \\
& \sum_{m \geq n \geq 0} u^{m} v^{n} f_{m, n}\left(z_{1}, z_{2} \mid q\right)=\sum_{j=0}^{\infty}\left(u z_{1}\right)^{j} g_{j}\left(z_{1} z_{2}, u v\right) .
\end{aligned}
$$

Theorem 2.12. Assume that

$$
w_{\alpha+\beta}(x)=x^{\alpha} w_{\beta}(x)
$$

and

$$
\phi_{n}(x ; \alpha)=\frac{1}{w_{\alpha}(x)} \partial_{x}^{n}\left(w_{\alpha}(x) x^{n}\right), \quad n=0,1, \ldots
$$

Then for $m \geq n$ we have

$$
\begin{aligned}
& f_{m, n}\left(z_{1}, z_{2} ; \beta\right)=\left(\frac{z_{1}}{z_{2}}\right)^{m-n} \frac{\partial_{z_{2}}^{n}\left(w_{\beta}\left(z_{1} z_{2}\right) z_{2}^{m}\right)}{w_{\beta}\left(z_{1} z_{2}\right)}, \\
& f_{m+1, n+1}\left(z_{1}, z_{2} ; \beta\right)=\frac{\partial_{z_{2}}\left(\left(\frac{z_{2}}{z_{1}}\right)^{m+1-n} w_{\beta}\left(z_{1} z_{2}\right) f_{m+1, n}\left(z_{1}, z_{2} ; \beta\right)\right)}{\left(\frac{z_{2}}{z_{1}}\right)^{m-n} w_{\beta}\left(z_{1} z_{2}\right)}, \\
& f_{m, n}\left(z_{1}, z_{2} ; \beta\right)=\frac{\partial_{z_{1}}^{n}\left(w_{\beta}\left(z_{1} z_{2}\right) z_{1}^{m}\right)}{w_{\beta}\left(z_{1} z_{2}\right)}, \\
& f_{m+1, n+1}\left(z_{1}, z_{2} ; \beta\right)=\frac{\partial_{z_{1}}\left(w_{\beta}\left(z_{1} z_{2}\right) f_{m+1, n}\left(z_{1}, z_{2} ; \beta\right)\right)}{w_{\beta}\left(z_{1} z_{2}\right)}
\end{aligned}
$$

and

$$
\partial_{z_{2}}\left(\left(\frac{z_{2}}{z_{1}}\right)^{m+1-n} w_{\beta}\left(z_{1} z_{2}\right) f_{m+1, n}\left(z_{1}, z_{2} ; \beta\right)\right)=\partial_{z_{1}}\left(w_{\beta}\left(z_{1} z_{2}\right) f_{m+1, n}\left(z_{1}, z_{2} ; \beta\right)\right) .
$$

Furthermore, if for some nonnegative integers $s, t$ such that

$$
\frac{w_{\beta}^{\prime}(x)}{w_{\beta}(x)}=\frac{\sum_{j=0}^{s} a_{j} x^{j}}{\sum_{j=0}^{t} b_{j} x^{j}},
$$


where $\left\{a_{j}\right\}_{j=0}^{s}$ and $\left\{b_{j}\right\}_{j=0}^{t}$ are certain real numbers. Then form $m \geq n$ we have

$$
\begin{aligned}
& \sum_{j=0}^{t} b_{j} z_{2}^{j} \frac{\partial_{z_{2}}\left(w_{\beta}\left(z_{1} z_{2}\right) f_{m+j-1, n}\left(z_{1}, z_{2} ; \beta\right)\right)}{w_{\beta}\left(z_{1} z_{2}\right)}=\sum_{j=0}^{s} a_{j} z_{2}^{j} f_{m+j, n}\left(z_{1}, z_{2} ; \beta\right), \\
& \sum_{j=0}^{t} b_{j} \frac{\partial_{z_{1}}\left(z_{1}^{n-m-j+1} w_{\beta}\left(z_{1} z_{2}\right) f_{m+j-1, n}\left(z_{1}, z_{2} ; \beta\right)\right)}{z_{1}^{n-m-j} z_{2}^{1-j} w_{\beta}\left(z_{1} z_{2}\right)}=\sum_{j=0}^{s} a_{j} z_{2}^{j} f_{m+j, n}\left(z_{1}, z_{2} ; \beta\right)
\end{aligned}
$$

and

$$
\begin{aligned}
\sum_{j=0}^{t} b_{j} & z_{2}^{j} \frac{\partial_{z_{2}}\left(w_{\beta}\left(z_{1} z_{2}\right) f_{m+j-1, n}\left(z_{1}, z_{2} ; \beta\right)\right)}{w_{\beta}\left(z_{1} z_{2}\right)} \\
& =\sum_{j=0}^{t} b_{j} \frac{\partial_{z_{1}}\left(z_{1}^{n-m-j+1} w_{\beta}\left(z_{1} z_{2}\right) f_{m+j-1, n}\left(z_{1}, z_{2} ; \beta\right)\right)}{z_{1}^{n-m-j} z_{2}^{1-j} w_{\beta}\left(z_{1} z_{2}\right)} .
\end{aligned}
$$

Proof. From

$$
\begin{aligned}
\partial_{z_{2}}^{n}\left[w_{\alpha}\left(z_{1} z_{2}\right)\left(z_{1} z_{2}\right)^{n}\right] & =\partial_{z_{2}}^{n}\left(w_{\beta}\left(z_{1} z_{2}\right)\left(z_{1} z_{2}\right)^{m}\right)=z_{1}^{m} \partial_{z_{2}}^{n}\left(w_{\beta}\left(z_{1} z_{2}\right) z_{2}^{m}\right) \\
& =z_{1}^{n} w_{\alpha}\left(z_{1} z_{2}\right) \phi_{n}\left(z_{1} z_{2} ; \alpha\right)=z_{1}^{n} z_{2}^{m-n} w_{\beta}\left(z_{1} z_{2}\right) f_{m, n}\left(z_{1}, z_{2} ; \beta\right)
\end{aligned}
$$

and

$$
\begin{aligned}
\partial_{z_{2}}[ & \left.\left(\frac{z_{2}}{z_{1}}\right)^{m+1-n} w_{\beta}\left(z_{1} z_{2}\right) f_{m+1, n}\left(z_{1}, z_{2} ; \beta\right)\right] \\
& =\partial_{z_{2}}^{n+1}\left(w_{\beta}\left(z_{1} z_{2}\right) z_{2}^{m+1}\right)=\left(\frac{z_{2}}{z_{1}}\right)^{m-n} w_{\beta}\left(z_{1} z_{2}\right) f_{m+1, n+1}\left(z_{1}, z_{2} ; \beta\right)
\end{aligned}
$$

to get (2.22) and (2.23), (2.24) and (2.25) are proved similarly. Equation (2.26) is obtained from (2.23) and (2.25).

From

$$
w_{\beta}\left(z_{1} z_{2}\right) f_{m, n}\left(z_{1}, z_{2} ; \beta\right)=\partial_{z_{1}}^{n}\left(w_{\beta}\left(z_{1} z_{2}\right) z_{1}^{m}\right)
$$

we get

$$
\partial_{z_{2}}\left(w_{\beta}\left(z_{1} z_{2}\right) f_{m, n}\left(z_{1}, z_{2} ; \beta\right)\right)=\partial_{z_{1}}^{n}\left(w_{\beta}^{(1)}\left(z_{1} z_{2}\right) z_{1}^{m+1}\right) .
$$

On the other hand

$$
\sum_{j=0}^{t} b_{j} z_{1}^{j+m} z_{2}^{j} w_{\beta}^{(1)}\left(z_{1} z_{2}\right)=\sum_{j=0}^{s} a_{j} z_{1}^{j+m} z_{2}^{j} w_{\beta}\left(z_{1} z_{2}\right)
$$

implies

$$
\sum_{j=0}^{t} b_{j} z_{2}^{j} \partial_{z_{1}}^{n}\left(z_{1}^{j+m} w_{\beta}^{(1)}\left(z_{1} z_{2}\right)\right)=\sum_{j=0}^{s} a_{j} z_{2}^{j} \partial_{z_{1}}^{n}\left(z_{1}^{j+m} w_{\beta}\left(z_{1} z_{2}\right)\right)
$$

which gives (2.27). Equation (2.28) is proved similarly. (2.29) is obtained from (2.27) and (2.28). 
The following theorem can be proved similarly.

Theorem 2.13. Assume that

$$
w_{\alpha+\beta}(x)=w_{\alpha+\beta}(x \mid q)=x^{\alpha} w_{\beta}(x \mid q)
$$

and

$$
\phi_{n}(x ; \alpha)=\phi_{n}(x ; \alpha \mid q)=\frac{1}{w_{\alpha}(x)} D_{q, x}^{n}\left(w_{\alpha}(x \mid q) x^{n}\right), \quad n=0,1, \ldots
$$

Let $f_{m, n}\left(z_{1}, z_{2} ; \beta \mid q\right)=f_{m, n}\left(z_{1}, z_{2} ; \beta\right)$, then for $m \geq n$ we have

$$
\begin{aligned}
& f_{m, n}\left(z_{1}, z_{2} ; \beta \mid q\right)=\frac{\left(\frac{z_{1}}{z_{2}}\right)^{m-n} \partial_{q, z_{2}}^{n}\left(w_{\beta}\left(z_{1} z_{2} \mid q\right) z_{2}^{m}\right)}{w_{\beta}\left(z_{1} z_{2} \mid q\right)}, \\
& f_{m+1, n+1}\left(z_{1}, z_{2} ; \beta \mid q\right)=\frac{\partial_{q, z_{2}}\left(\left(\frac{z_{2}}{z_{1}}\right)^{m+1-n} w_{\beta}\left(z_{1} z_{2} \mid q\right) f_{m+1, n}\left(z_{1}, z_{2} ; \beta \mid q\right)\right)}{\left(\frac{z_{2}}{z_{1}}\right)^{m-n} w_{\beta}\left(z_{1} z_{2} \mid q\right)} \\
& f_{m, n}\left(z_{1}, z_{2} ; \beta \mid q\right)=\frac{\partial_{q, z_{1}}^{n}\left(w_{\beta}\left(z_{1} z_{2} \mid q\right) z_{1}^{m}\right)}{w_{\beta}\left(z_{1} z_{2} \mid q\right)}, \\
& f_{m+1, n+1}\left(z_{1}, z_{2} ; \beta \mid q\right)=\frac{\partial_{q, z_{1}}\left(w_{\beta}\left(z_{1} z_{2} \mid q\right) f_{m+1, n}\left(z_{1}, z_{2} ; \beta \mid q\right)\right)}{w_{\beta}\left(z_{1} z_{2} \mid q\right)}
\end{aligned}
$$

and

$$
\begin{aligned}
\partial_{q, z_{2}} & \left(\left(\frac{z_{2}}{z_{1}}\right)^{m+1-n} w_{\beta}\left(z_{1} z_{2} \mid q\right) f_{m+1, n}\left(z_{1}, z_{2} ; \beta \mid q\right)\right) \\
= & \left(\frac{z_{2}}{z_{1}}\right)^{m-n} \partial_{q, z_{1}}\left(w_{\beta}\left(z_{1} z_{2} \mid q\right) f_{m+1, n}\left(z_{1}, z_{2} ; \beta \mid q\right)\right) .
\end{aligned}
$$

Furthermore, if for some nonnegative integers $s, t$ and real numbers $\left\{a_{j}\right\}_{j=0}^{s}$ and $\left\{b_{j}\right\}_{j=0}^{t}$ we have

$$
\frac{D_{q, x} w_{\beta}(x \mid q)}{w_{\beta}(x \mid q)}=\frac{\sum_{j=0}^{s} a_{j} x^{j}}{\sum_{j=0}^{t} b_{j} x^{j}},
$$

then for $m \geq n$

$$
\begin{aligned}
& \sum_{j=0}^{t} b_{j} \frac{\partial_{q, z_{1}}\left(z_{1}^{n-m-j+1} w_{\beta}\left(z_{1} z_{2} \mid q\right) f_{m+j-1, n}\left(z_{1}, z_{2} ; \beta \mid q\right)\right)}{z_{1}^{n-m-j} z_{2}^{1-j} w_{\beta}\left(z_{1} z_{2} \mid q\right)}=\sum_{j=0}^{s} a_{j} z_{2}^{j} f_{m+j, n}\left(z_{1}, z_{2} ; \beta \mid q\right), \\
& \sum_{j=0}^{t} b_{j} z_{2}^{j} \frac{\partial_{q, z_{2}}\left(w_{\beta}\left(z_{1} z_{2} \mid q\right) f_{m+j-1, n}\left(z_{1}, z_{2} ; \beta \mid q\right)\right)}{w_{\beta}\left(z_{1} z_{2} \mid q\right)}=\sum_{j=0}^{s} a_{j} z_{2}^{j} f_{m+j, n}\left(z_{1}, z_{2} ; \beta \mid q\right)
\end{aligned}
$$

and

$$
\begin{aligned}
& \sum_{j=0}^{t} b_{j} \frac{\partial_{q, z_{1}}\left(z_{1}^{n-m-j+1} w_{\beta}\left(z_{1} z_{2} \mid q\right) f_{m+j-1, n}\left(z_{1}, z_{2} ; \beta \mid q\right)\right)}{z_{1}^{n-m-j} z_{2}^{1-j} w_{\beta}\left(z_{1} z_{2} \mid q\right)} \\
& \quad=\sum_{j=0}^{t} b_{j} z_{2}^{j} \frac{\partial_{q, z_{2}}\left(w_{\beta}\left(z_{1} z_{2} \mid q\right) f_{m+j-1, n}\left(z_{1}, z_{2} ; \beta \mid q\right)\right)}{w_{\beta}\left(z_{1} z_{2} \mid q\right)} .
\end{aligned}
$$


We now discuss monotonicity of zeros of $\left\{f_{m, n}(z, \bar{z})\right\}$. The following version of Markov's theorem is Theorem 7.1.1 in [17].

Theorem 2.14. Let $\left\{p_{n}(x ; \tau)\right\}$ be orthogonal with respect to $d \alpha(x ; \tau)$,

$$
d \mu(x ; \tau)=\rho(x ; \tau) d \beta(x),
$$

on an interval $I=(a, b)$ and assume that $\rho(x ; \tau)$ is positive and has a continuous first derivative with respect to $\tau$ for $x \in I, \tau \in T=\left(\tau_{1}, \tau_{2}\right)$. Furthermore, assume that

$$
\int_{a}^{b} x^{j} \rho_{\tau}(x ; \tau) d \beta(x), \quad j=0,1, \ldots, 2 n-1,
$$

converge uniformly for $\tau$ in every compact subinterval of $T$. Then the zeros of the orthogonal polynomials $p_{n}(x ; \tau)$ are increasing (decreasing) functions of $\tau, \tau \in T$, if $\partial\{\ln \rho(x ; \tau)\} / \partial \tau$ is an increasing (decreasing) function of $x, x \in I$.

The standard version of Markov's theorem is when $\beta(x)=x$, see [31].

By applying Theorem 2.14 to the polynomials $\left\{\phi_{n}(r ; \alpha)\right\}$ defined in (2.1) it follows that the zeros of $\phi_{n}(r ; \alpha)$ increase with $\alpha, \alpha \geq 0$. Now fix $n$ and assume that $m \geq n$. In view of the definition of the polynomials $\left\{f_{m, n}(z, \bar{z})\right\}, m \geq n$, in (2.2) we see that if $r_{0}$ is a zero of $\phi_{n}(r ; \alpha)$ then $f_{m, n}(z, \bar{z})=0$ for all $z$ on the circle $|z|=\sqrt{r_{0}}$. Since $\alpha=m-n$ the following theorem holds.

Theorem 2.15. For fixed $n$ the radii of the circles forming the zero sets of $f_{m, n}(z, \bar{z})$ increase with $m$ for all $m \geq n$.

\section{The polynomials $\left\{Z_{m, n}^{(\beta)}\left(z_{1}, z_{2}\right)\right\}$}

Motivated by the class of general $1 D$ systems in Section 2 we define polynomials $\left\{Z_{m, n}^{(\beta)}\left(z_{1}, z_{2}\right)\right\}$ by

$$
Z_{m, n}^{(\beta)}\left(z_{1}, z_{2}\right)=\frac{1}{n !} \sum_{k=0}^{n}\left(\begin{array}{l}
n \\
k
\end{array}\right) \frac{(\beta+1)_{m}}{(\beta+1)_{m-k}}(-1)^{n-k} z_{1}^{m-k} z_{2}^{n-k}
$$

for $m \geq n$. When $m<n$ the polynomials are defined by

$$
Z_{m, n}^{(\beta)}\left(z_{1}, z_{2}\right)=Z_{n, m}^{(\beta)}\left(z_{2}, z_{1}\right)
$$

Here $\beta>-1$.

These polynomials arise through the choice $\phi_{n}(x ; \alpha)=L_{n}^{(\alpha+\beta)}(x)$, where $L_{n}^{(a)}(x)$ is a Laguerre polynomial $[17,31]$

$$
L_{n}^{(a)}(x)=\frac{(a+1)_{n}}{n !} \sum_{k=0}^{n}\left(\begin{array}{l}
n \\
k
\end{array}\right) \frac{(-x)^{k}}{(a+1)_{k}}=\frac{(a+1)_{n}}{n !} \sum_{k=0}^{n}\left(\begin{array}{l}
n \\
k
\end{array}\right) \frac{(-x)^{n-k}}{(a+1)_{n-k}} .
$$

Indeed, for $m \geq n$

$$
Z_{m, n}^{(\beta)}\left(z_{1}, z_{2}\right)=z_{1}^{m-n} L_{n}^{(\beta+m-n)}\left(z_{1} z_{2}\right)
$$

and

$$
z_{1} Z_{m, n}^{(\beta+1)}\left(z_{1}, z_{2}\right)=Z_{m+1, n}^{(\beta)}\left(z_{1}, z_{2}\right)
$$


It is clear that when $\beta=0$ we see that

$$
H_{m, n}\left(z_{1}, z_{2}\right)=(-1)^{n}(m \wedge n) ! Z_{m, n}^{(0)}\left(z_{1}, z_{2}\right) .
$$

Therefore in the notation of Section 2, we have

$$
\zeta_{n}(\alpha)=\frac{\Gamma(\alpha+\beta+n+1)}{n !}, \quad c_{j}(n, \alpha)=\frac{(-1)^{n-j}(\alpha+\beta+1)_{n}}{(n-j) ! j !(\alpha+\beta+1)_{n-j}} .
$$

Theorem 3.1. For $m, n, s, t=0,1, \ldots$ and $\beta>-1$, we have the orthogonality relation

$$
\int_{\mathbb{R}^{2}} Z_{m, n}^{(\beta)}(z, \bar{z}) \overline{Z_{s, t}^{(\beta)}(z, \bar{z})}(z \bar{z})^{\beta} e^{-z \bar{z}} r d r d \theta=\pi \frac{\Gamma(\beta+m \vee n+1)}{(m \wedge n) !} \delta_{m, s} \delta_{n, t} .
$$

This follows from Theorem 2.1 in the case (3.2) but for completeness we give a direct proof. Alternate proof. When $m \geq n$ we consider the integral

$$
I(m, n, a, b):=\int_{\mathbb{R}^{2}} Z_{m, n}^{(\beta)}(z, \bar{z}) \overline{\left(z^{a} \bar{z}^{b}\right)} r^{2 \beta+1} e^{-r^{2}} d r d \theta,
$$

for $a \leq m, b \leq n$. Therefore

$$
\begin{aligned}
I(m, n, a, b) & :=2 \pi \delta_{b+m, a+n} \frac{(-1)^{n}}{n !} \sum_{k=0}^{n}\left(\begin{array}{l}
n \\
k
\end{array}\right) \frac{(\beta+1)_{m}}{(\beta+1)_{m-k}}(-1)^{k} \int_{0}^{\infty} r^{a+b+m+n-2 k+2 \beta+1} e^{-r^{2}} d r \\
& =\pi \delta_{b+m, a+n} \frac{(\beta+1)_{m}}{n !} \sum_{k=0}^{n}\left(\begin{array}{l}
n \\
k
\end{array}\right) \frac{(-1)^{n-k}}{(\beta+1)_{m-k}} \Gamma(1+\beta-k+(a+b+m+n) / 2) \\
& =\pi \delta_{b+m, a+n} \frac{(\beta+1)_{m}}{n !} \sum_{k=0}^{n}\left(\begin{array}{l}
n \\
k
\end{array}\right) \frac{(-1)^{n-k}}{(\beta+1)_{m-k}} \Gamma(1+\beta-k+a+n) \\
& =\pi \delta_{b+m, a+n} \frac{\Gamma(1+\beta+a)}{(\beta+1)_{m-n}} \frac{(\beta+1)_{m}}{n !}{ }_{2} F_{1}(-n, 1+a+\beta ; \beta+1+m-n ; 1) \\
& =\pi \delta_{b+m, a+n} \frac{\Gamma(1+\beta+a)}{(\beta+1)_{m-n}} \frac{(\beta+1)_{m}}{n !} \frac{(m-n-a)_{n}}{(\beta+1+m-n)_{n}} .
\end{aligned}
$$

Since $m-n-a=-b$ we that the integral vanishes for $b<n$. If $b=n$ then $a=m$ and we conclude that

$$
\int_{\mathbb{R}^{2}} Z_{m, n}^{(\beta)}(z, \bar{z}) \overline{\left(z^{a} \bar{z}^{b}\right)} r^{2 \beta+1} e^{-r^{2}} d r d \theta=\pi \Gamma(1+\beta+m) \delta_{a, m} \delta_{b, n},
$$

and the theorem now follows.

In view of (3.2) the recurrence relations (2.7), (2.5) and (2.12) become

$$
\begin{aligned}
& z_{1} Z_{m, n}^{(\beta)}\left(z_{1}, z_{2}\right)=Z_{m+1, n}^{(\beta)}\left(z_{1}, z_{2}\right)-Z_{m, n-1}^{(\beta)}\left(z_{1}, z_{2}\right), \\
& z_{2} Z_{m+1, n}^{(\beta)}\left(z_{1}, z_{2}\right)=-(n+1) Z_{m+1, n+1}^{(\beta)}\left(z_{1}, z_{2}\right)+(\beta+m+1) Z_{m, n}^{(\beta)}\left(z_{1}, z_{2}\right),
\end{aligned}
$$

and

$$
\begin{aligned}
(\beta+ & \left.m+n+1-z_{1} z_{2}\right) Z_{m, n}^{(\beta)}\left(z_{1}, z_{2}\right) \\
& =(n+1) Z_{m+1, n+1}^{(\beta)}\left(z_{1}, z_{2}\right)+(m+\beta) Z_{m-1, n-1}^{(\beta)}\left(z_{1}, z_{2}\right)
\end{aligned}
$$

respectively, where $m \geq n$. We now discuss differential recurrence relations. 
Theorem 3.2. For $m \geq n$, the polynomials $\left\{Z_{m, n}^{(\beta)}\left(z_{1}, z_{2}\right)\right\}$ satisfy the differential recurrence relations

$$
\begin{aligned}
& \delta_{z_{2}} Z_{m, n}^{(\beta)}\left(z_{1}, z_{2}\right)=-z_{2} Z_{m, n-1}^{(\beta)}\left(z_{1}, z_{2}\right), \\
& \delta_{z_{2}} Z_{m, n}^{(\beta)}\left(z_{1}, z_{2}\right)=n Z_{m, n}^{(\beta)}\left(z_{1}, z_{2}\right)-(\beta+m) Z_{m-1, n-1}^{(\beta)}\left(z_{1}, z_{2}\right), \\
& \delta_{z_{1}} Z_{m, n}^{(\beta)}\left(z_{1}, z_{2}\right)=(m-n) Z_{m, n}^{(\beta)}\left(z_{1}, z_{2}\right)-z_{2} Z_{m, n-1}^{(\beta)}\left(z_{1}, z_{2}\right), \\
& \delta_{z_{1}} Z_{m, n}^{(\beta)}\left(z_{1}, z_{2}\right)=m Z_{m, n}^{(\beta)}\left(z_{1}, z_{2}\right)-(m+\beta) Z_{m-1, n-1}^{(\beta)}\left(z_{1}, z_{2}\right), \\
& \left(\delta_{z_{1}}-\delta_{z_{2}}\right) Z_{m, n}^{(\beta)}\left(z_{1}, z_{2}\right)=(m-n) Z_{m, n}^{(\beta)}\left(z_{1}, z_{2}\right), \\
& \left(n \delta_{z_{1}}-m \delta_{z_{2}}\right) Z_{m, n}^{(\beta)}\left(z_{1}, z_{2}\right)=(m-n)(m+\beta) Z_{m-1, n-1}^{(\beta)}\left(z_{1}, z_{2}\right),
\end{aligned}
$$

where $\delta_{z_{1}}=z_{1} \partial_{z_{1}}, \delta_{z_{2}}=z_{2} \partial_{z_{2}}$. Moreover they have the operational representation

$$
Z_{m, n}^{(\beta)}\left(z_{1}, z_{2}\right)=\frac{(-1)^{n}}{n !} z_{1}^{-\beta} \exp \left(-\partial_{z_{1}} \partial_{z_{2}}\right) z_{1}^{\beta+m} z_{2}^{n}
$$

and the Rodrigues type representation

$$
\begin{aligned}
Z_{m, n}^{(\beta)}\left(z_{1}, z_{2}\right) & =\frac{1}{n !} z_{1}^{-\beta} e^{z_{1} z_{2}} \partial_{z_{1}}^{n}\left(z_{1}^{m+\beta} e^{-z_{1} z_{2}}\right) \\
& =\frac{(-1)^{m}}{n !}\left(z_{1} z_{2}\right)^{-\beta} e^{z_{1} z_{2}} \partial_{z_{1}}^{n}\left(\left(z_{1} z_{2}\right)^{\beta} \partial_{z_{2}}^{m}\left(e^{-z_{1} z_{2}}\right)\right) .
\end{aligned}
$$

Proof. Observe that from (3.1) we obtain

$$
\partial_{z_{2}} Z_{m, n}^{(\beta)}\left(z_{1}, z_{2}\right)=\sum_{k=0}^{n} \frac{(-1)^{n-k}}{k !(n-k) !} \frac{(\beta+1)_{m}}{(\beta+1)_{m-k}} z_{1}^{m-k}(n-k) z_{2}^{n-k-1} .
$$

Therefore we have

$$
\partial_{z_{2}} Z_{m, n}^{(\beta)}\left(z_{1}, z_{2}\right)=-\sum_{k=0}^{n-1} \frac{(-1)^{n-1-k}}{k !(n-1-k) !} \frac{(\beta+1)_{m}}{(\beta+1)_{m-k}} z_{1}^{m-k} z_{2}^{n-k-1}=-Z_{m, n-1}^{(\beta)}\left(z_{1}, z_{2}\right)
$$

and

$$
\begin{aligned}
\partial_{z_{2}} Z_{m, n}^{(\beta)}\left(z_{1}, z_{2}\right)= & \frac{n}{z_{2}} \sum_{k=0}^{n} \frac{(-1)^{n-k}}{k !(n-k) !} \frac{(\beta+1)_{m}}{(\beta+1)_{m-k}} z_{1}^{m-k} z_{2}^{n-k} \\
& -\sum_{k=1}^{n} \frac{(-1)^{n-k}}{(k-1) !(n-k) !} \frac{(\beta+1)_{m}}{(\beta+1)_{m-k}} z_{1}^{m-k} z_{2}^{n-k-1} \\
= & \frac{n}{z_{2}} Z_{m, n}^{(\beta)}\left(z_{1}, z_{2}\right)+\sum_{j=0}^{n-1} \frac{(-1)^{n-j}}{j !(n-1-j) !} \frac{(\beta+1)_{m} z_{1}^{m-1-j} z_{2}^{n-j-2}}{(\beta+1)_{m-1-j}} \\
= & \frac{n}{z_{2}} Z_{m, n}^{(\beta)}\left(z_{1}, z_{2}\right)-\frac{(\beta+m)}{z_{2}} \sum_{j=0}^{n-1} \frac{(-1)^{n-1-j}}{j !(n-1-j) !} \frac{(\beta+1)_{m-1} z_{1}^{m-1-j} z_{2}^{n-1-j}}{(\beta+1)_{m-1-j}} \\
= & \frac{n}{z_{2}} Z_{m, n}^{(\beta)}\left(z_{1}, z_{2}\right)-\frac{(\beta+m)}{z_{2}} Z_{m-1, n-1}^{(\beta)}\left(z_{1}, z_{2}\right) .
\end{aligned}
$$

The relationships (3.7) and (3.8) are proved similarly. Formulas (3.11) and (3.12) follow by direct calculation using (3.1). The first-order partial differential equation (3.9) can be obtained from either (3.5) and (3.7) or (3.6) and (3.8), (3.10) is proved similarly. 
One would have expected the Rodrigues formula for $Z_{m, n}^{(\beta)}\left(z_{1}, z_{2}\right)$ to be a constant multiple of

$$
\left(z_{1} z_{2}\right)^{-\beta} e^{z_{1} z_{2}} \partial_{z_{1}}^{n} \partial_{z_{2}}^{m}\left(\left(z_{1} z_{2}\right)^{\beta} e^{-z_{1} z_{2}}\right)
$$

which is obviously false except in the $2 D$-Hermite case $\beta=0$.

Note that (3.4) follows from (3.12) by writing $\partial_{z_{1}}^{n}$ as $\partial_{z_{1}}^{n-1} \partial_{z_{1}}$.

We shall use the notation

$$
w_{\beta}\left(z_{1} z_{2}\right)=\left(z_{1} z_{2}\right)^{\beta} \exp \left(-z_{1} z_{2}\right) .
$$

It is clear that (3.12) implies the differentiation formulas

$$
e^{z_{1} z_{2}} \partial_{z_{2}}\left(e^{-z_{1} z_{2}} Z_{m, n}^{(\beta)}\left(z_{1}, z_{2}\right)\right)=-z_{1} Z_{m, n}^{(\beta+1)}\left(z_{1}, z_{2}\right)=-Z_{m+1, n}^{(\beta)}\left(z_{1}, z_{2}\right)
$$

and

$$
\frac{1}{w_{\beta}\left(z_{1} z_{2}\right)} \partial_{z_{1}}\left(w_{\beta}\left(z_{1} z_{2}\right) Z_{m, n}^{(\beta)}\left(z_{1}, z_{2}\right)\right)=(n+1) Z_{m, n+1}^{(\beta)}\left(z_{1}, z_{2}\right)
$$

for $m \geq n$. It also clear that

$$
\delta_{z_{j}} w_{\beta}\left(z_{1} z_{2}\right)=\left(\beta-z_{1} z_{2}\right) w_{\beta}\left(z_{1} z_{2}\right), \quad j=1,2
$$

and (3.3) which imply the relationships

$$
\frac{\delta_{z_{2}}\left(w_{\beta}\left(z_{1} z_{2}\right) Z_{m, n}^{(\beta)}\left(z_{1}, z_{2}\right)\right)}{w_{\beta}\left(z_{1} z_{2}\right)}=\beta Z_{m, n}^{(\beta)}\left(z_{1}, z_{2}\right)-z_{2} Z_{m+1, n}^{(\beta)}\left(z_{1}, z_{2}\right),
$$

and

$$
\frac{\delta_{z_{1}}\left(w_{\beta}\left(z_{1} z_{2}\right) Z_{m, n}^{(\beta)}\left(z_{1}, z_{2}\right)\right)}{w_{\beta}\left(z_{1} z_{2}\right)}=(\beta+m-n) Z_{m, n}^{(\beta)}\left(z_{1}, z_{2}\right)-z_{2} Z_{m+1, n}^{(\beta)}\left(z_{1}, z_{2}\right) .
$$

This can be seen by first applying (3.14), then applying (3.3), for example,

$$
\begin{aligned}
& \frac{1}{w_{\beta}\left(z_{1} z_{2}\right)} \partial_{z_{2}}\left(w_{\beta}\left(z_{1} z_{2}\right) Z_{m, n}^{(\beta)}\left(z_{1}, z_{2}\right)\right) \\
& \quad=\frac{\beta-z_{1} z_{2}}{z_{2}} Z_{m, n}^{(\beta)}\left(z_{1}, z_{2}\right)-Z_{m, n-1}^{(\beta)}\left(z_{1}, z_{2}\right)=\frac{\beta}{z_{2}} Z_{m, n}^{(\beta)}\left(z_{1}, z_{2}\right)-Z_{m+1, n}^{(\beta)}\left(z_{1}, z_{2}\right) .
\end{aligned}
$$

Theorem 3.3. The polynomials $\left\{Z_{m, n}^{(\beta)}\left(z_{1}, z_{2}\right)\right\}$ satisfy the second-order partial differential equation

$$
\partial_{\partial z_{1}} \partial_{\partial z_{2}} f+\left(\frac{\beta-z_{1} z_{2}}{z_{1}}\right) \partial_{\partial z_{2}} f=-n f
$$

for all $m \geq n$.

Proof. From equations (3.13), (3.14) and (3.5) we get

$$
\begin{aligned}
& \frac{\partial_{z_{1}} w_{\beta}\left(z_{1} z_{2}\right) Z_{m, n}^{(\beta)}\left(z_{1}, z_{2}\right)+w_{\beta}\left(z_{1} z_{2}\right) \partial_{z_{1}} Z_{m, n}^{(\beta)}\left(z_{1}, z_{2}\right)}{w_{\beta}\left(z_{1} z_{2}\right)} \\
& \quad=\left(\frac{\beta}{z_{1}}-z_{2}\right) Z_{m, n}^{(\beta)}\left(z_{1}, z_{2}\right)+\partial_{z_{1}} Z_{m, n}^{(\beta)}\left(z_{1}, z_{2}\right)=(n+1) Z_{m, n+1}^{(\beta)}\left(z_{1}, z_{2}\right)
\end{aligned}
$$

and

$$
\begin{aligned}
\partial_{z_{2}}\{ & \left.\left(\frac{\beta}{z_{1}}-z_{2}\right) Z_{m, n}^{(\beta)}\left(z_{1}, z_{2}\right)\right\}+\partial_{z_{1}} \partial_{z_{2}} Z_{m, n}^{(\beta)}\left(z_{1}, z_{2}\right) \\
& =(n+1) \partial_{z_{2}} Z_{m, n+1}^{(\beta)}\left(z_{1}, z_{2}\right)=-(n+1) Z_{m, n}^{(\beta)}\left(z_{1}, z_{2}\right),
\end{aligned}
$$

which simplifies to the desired second-order partial differential equation. 
It is clear that the differential property $(3.15)$ can be written in the form

$$
\frac{\partial}{\partial z_{1}}\left[w_{\beta}\left(z_{1} z_{2}\right) \frac{\partial}{\partial z_{2}} Z_{m, n}^{(\beta)}\left(z_{1}, z_{2}\right)\right]=-n Z_{m, n}^{(\beta)}\left(z_{1}, z_{2}\right) .
$$

Note that (3.15) and (3.9) indicate that the polynomials $\left\{Z_{m, n}^{(\beta)}\left(z_{1}, z_{2}\right)\right\}$ are simultaneous eigenfunctions of the operators on their respective left-hand sides. The differential operators $\partial_{\partial z_{1}} \partial_{\partial z_{2}}+\left(\frac{\beta-z_{1} z_{2}}{z_{1}}\right) \partial_{\partial z_{2}}$ and $z_{1} \partial_{\partial z_{1}}-z_{2} \partial_{\partial z_{2}}$ indeed commute as can be directly verified.

Let $w(x, y)=(z \bar{z})^{\beta} e^{-z \bar{z}}=r^{2 \beta+1} e^{-r^{2}} d r d \theta$. It is clear that the bilinear functional

$$
\langle f, g\rangle=\int_{\mathbb{R}^{2}} f(x, y) \overline{g(x, y)} w(x, y) d x d y
$$

defines a semi-inner product on the linear space of $w(x, y) d x d y$ measurable functions $f(x, y)$ such that $\int_{\mathbb{R}^{2}}|f(x, y)|^{2} w(x, y) d x d y<\infty$. Since a polynomial $p(x, y)$ is also a polynomial in $r$ with trigonometric polynomials as coefficients, hence it is a continuous function in $(r, \theta)$. Then $\int_{\mathbb{R}^{2}}|p(x, y)|^{2} w(x, y) d x d y=0$ implies that $p(x, y)=0$, consequently the bilinear functional is positive definite on the subspace of all the polynomials $p(x, y)$. In the subsequent discussion we only need this inner product space of all the polynomials.

Theorem 3.4. We have the adjoint relations

$$
\left(\partial_{z}\right)^{*}=-\partial_{\bar{z}}-\frac{\beta}{\bar{z}}+z, \quad\left(\partial_{\bar{z}}\right)^{*}=-\partial_{z}-\frac{\beta}{z}+\bar{z} .
$$

The proof is straightforward calculus exercise.

This allows us to write (3.15) or (3.16) in the selfadjoint form

$$
\left(\left(\partial_{\bar{z}}\right)^{*} \partial_{\bar{z}}\right) f=n f .
$$

Moreover we also have the following result.

Theorem 3.5. The operator $A:=\left(\partial_{\bar{z}}\right)^{*} \partial_{\bar{z}}$ is positive in the sense that $\langle A f, f\rangle \geq 0$ with $=$ if and only if $\partial_{\bar{z}} f=0$, that is $f$ depends only on $z$.

This indicates that

$$
\frac{1}{w(x, y)} \frac{\partial}{\partial z} Z_{m, n}^{(\beta)}(z, \bar{z})=(n+1) Z_{m, n}^{(\beta)}(z, \bar{z}) .
$$

This is the adjoint of (3.5).

Ismail and Zeng [20] established the connection relations stated in the next theorem.

Theorem 3.6. We have the connection relation

$$
Z_{m, n}^{(\beta)}\left(z_{1}, z_{2}\right)=\sum_{j=0}^{n} \frac{(\beta-\gamma)_{j}}{j !}(-1)^{j} Z_{m-j, n-j}^{(\gamma)}\left(z_{1}, z_{2}\right), \quad m \geq n .
$$

For $m \geq n$, we have the special cases

$$
\begin{aligned}
& n ! Z_{m, n}^{(\beta)}\left(z_{1}, z_{2}\right)=\sum_{j=0}^{n}\left(\begin{array}{c}
n \\
j
\end{array}\right)(\beta)_{j}(-1)^{j} H_{m-j, n-j}\left(z_{1}, z_{2}\right), \\
& H_{m, n}\left(z_{1}, z_{2}\right)=n ! \sum_{j=0}^{n} \frac{(-\beta)_{j}}{j !}(-1)^{j} Z_{m-j, n-j}^{(\beta)}\left(z_{1}, z_{2}\right) .
\end{aligned}
$$


The Laguerre differential equation is [28, 31]

$$
x y^{\prime \prime}+(1+\alpha-x) y^{\prime}+n y=0 .
$$

Therefore Theorem 2.10 shows that

$$
z_{2} \frac{\partial^{2} Z_{m, n}^{(\beta)}}{\partial z_{2}^{2}}+\left(1+\beta+m-n-z_{1} z_{2}\right) \frac{\partial Z_{m, n}^{(\beta)}}{\partial z_{2}}+n z_{1} Z_{m, n}^{(\beta)}=0
$$

Ismail and Zeng [20] gave the generating function

$$
\sum_{m \geq n \geq 0} \frac{u^{m} v^{n}}{(m-n) !} Z_{m, n}^{(\beta)}\left(z_{1}, z_{2}\right)=(1+u v)^{-\beta-1} \exp \left(\frac{u v z_{1} z_{2}+z_{1} u}{1+u v}\right)
$$

this can be seen from

$$
g_{\alpha}(x, z)=\sum_{n=0}^{\infty} L_{n}^{(\alpha+\beta)}(x) z^{n}=\frac{\exp \left\{-\frac{x z}{1-z}\right\}}{(1-z)^{\alpha+\beta+1}},
$$

and $(2.21)$,

$$
\begin{aligned}
& \sum_{m \geq n \geq 0} \frac{u^{m} v^{n}}{(m-n) !} Z_{m, n}^{(\beta)}\left(z_{1}, z_{2}\right)=\frac{\exp \left\{\frac{u z_{1}-z_{1} z_{2} u v}{1-u v}\right\}}{(1-u v)^{\beta+1}}, \\
& \sum_{m \geq n \geq 0} u^{m} v^{n} Z_{m, n}^{(\beta)}\left(z_{1}, z_{2}\right)=\frac{\exp \left\{-\frac{z_{1} z_{2} u v}{1-u v}\right\}}{(1-u v)^{\beta}\left(1-z_{1} u-u v\right)} .
\end{aligned}
$$

It is clear that the generating function (3.17) implies the identity

$$
Z_{m, n}^{(\beta+\gamma+1)}\left(z_{1}+z_{3}, z_{2}+z_{4}\right)=\sum_{m \geq j \geq k \geq 0} \frac{Z_{j, k}^{(\beta)}\left(z_{1}, z_{2}\right) Z_{m-j, n-k}^{(\gamma)}\left(z_{3}, z_{4}\right)}{(j-k) !(m-n-j+k) !} .
$$

This is an analogue of the convolution identity

$$
L_{n}^{(\alpha+\beta+1)}(x+y)=\sum_{k=0}^{n} L_{k}^{(\alpha)}(x) L_{n}^{(\beta)}(y) .
$$

Al-Salam and Chihara characterized all 1- $D$ orthogonal polynomials satisfying convolution formulas in [1]. They discovered the Al-Salam-Chihara polynomials through this characterization [17]. It will be interesting to solve the corresponding 2- $D$ characterization problem.

\section{The polynomials $\left\{M_{n}^{(\beta, \gamma)}\left(z_{1}, z_{2}\right)\right\}$}

For $\alpha>0, \beta, \gamma>-1$ Ismail and Zeng [20] introduced the polynomials $\phi_{n}(r, \alpha)=P_{n}^{(\alpha+\gamma, \beta)}(1-2 r)$, that is

$$
\phi_{n}(r, \alpha)=(\alpha+\gamma+1)_{n} \sum_{k=0}^{n} \frac{(\alpha+\beta+\gamma+n+1)_{n-k}(-r)^{n-k}}{k !(n-k) !(\alpha+\gamma+1)_{n-k}} .
$$

They satisfy the following orthogonality relation

$$
\int_{0}^{1} \phi_{m}(r, \alpha) \phi_{n}(r, \alpha) u^{\alpha+\gamma}(1-u)^{\beta} d u=\zeta_{n}(\alpha+\gamma, \beta) \delta_{m, n}
$$


where

$$
\zeta_{n}(\alpha+\gamma, \beta)=\frac{\Gamma(\alpha+\gamma+n+1) \Gamma(\beta+n+1)}{n ! \Gamma(\alpha+\beta+\gamma+n+1)(\alpha+\beta+\gamma+2 n+1)} .
$$

We define the two variable polynomial $M_{m, n}^{(\beta, \gamma)}\left(z_{1}, z_{2}\right)$ by

$$
M_{m, n}^{(\beta, \gamma)}\left(z_{1}, z_{2}\right)=\sum_{k=0}^{n} \frac{(m+\beta+\gamma+1)_{n-k}(\gamma+1)_{m} z_{1}^{m-k}\left(-z_{2}\right)^{n-k}}{k !(n-k) !(\gamma+1)_{m-k}}
$$

for $m \geq n$ and

$$
M_{m, n}^{(\beta, \gamma)}\left(z_{1}, z_{2}\right)=M_{n, m}^{(\beta, \gamma)}\left(z_{2}, z_{1}\right), \quad m<n .
$$

Then

$$
z_{1} M_{m, n}^{(\beta, \gamma+1)}\left(z_{1}, z_{2}\right)=M_{m+1, n}^{(\beta, \gamma)}\left(z_{1}, z_{2}\right) .
$$

Clearly, $M_{m, n}^{(\beta, \gamma)}\left(z_{1}, z_{2}\right)$ satisfy the orthogonality relation

$$
\begin{aligned}
\int_{|z| \leq 1} & M_{m, n}^{(\beta, \gamma)}(z, \bar{z}) \overline{M_{p, q}^{(\beta, \gamma)}(z, \bar{z})} r^{2 \gamma}\left(1-r^{2}\right)^{\beta} 2 r d r \frac{d \theta}{2 \pi} \\
= & \frac{\Gamma(\gamma+m+1) \Gamma(\beta+n+1)}{n ! \Gamma(\beta+\gamma+m+1)(\beta+\gamma+m+n+1)} \delta_{m, p} \delta_{n, q}
\end{aligned}
$$

for $m \geq n$ as it is stated in [20]. The disk polynomials defined in [7] can be expressed as

$$
P_{m, n}^{\beta}(z)=\frac{(-1)^{n} n !}{(\beta+1)_{n}} M_{m, n}^{(\beta, 0)}(z, \bar{z}), \quad m \geq n .
$$

From (4.1) we get

$$
c_{k}(n, \alpha)=\frac{(m+\beta+\gamma+1)_{n-k}(\gamma+1)_{m}(-1)^{n-k}}{k !(n-k) !(\gamma+1)_{m-k}},
$$

where $\alpha=m-n$ with $m \geq n$. Then

$$
\begin{aligned}
& (\beta+\gamma+m+n+2) z_{2} M_{m+1, n}^{(\beta, \gamma)}\left(z_{1}, z_{2}\right) \\
& \quad=(\gamma+m+1) M_{m, n}^{(\beta, \gamma)}\left(z_{1}, z_{2}\right)-(n+1) M_{m+1, n+1}^{(\beta, \gamma)}\left(z_{1}, z_{2}\right), \\
& (\beta+\gamma+m+n+1) z_{1} M_{m, n}^{(\beta, \gamma)}\left(z_{1}, z_{2}\right) \\
& \quad=(\beta+\gamma+m+1) M_{m+1, n}^{(\beta, \gamma)}\left(z_{1}, z_{2}\right)-(\beta+n) M_{m, n-1}^{(\beta, \gamma)}\left(z_{1}, z_{2}\right)
\end{aligned}
$$

and

$$
\left(c_{n}-z_{1} z_{2}\right) M_{m, n}^{(\beta, \gamma)}\left(z_{1}, z_{2}\right)=a_{n} M_{m+1, n+1}^{(\beta, \gamma)}\left(z_{1}, z_{2}\right)+b_{n} M_{m-1, n-1}^{(\beta, \gamma)}\left(z_{1}, z_{2}\right),
$$

where

$$
\begin{aligned}
& a_{n}=\frac{(n+1)(\beta+\gamma+m+1)}{(\beta+\gamma+m+n+1)(\beta+\gamma+m+n+2)}, \\
& b_{n}=\frac{(\gamma+m)(\beta+n)}{(\beta+\gamma+m+n)(\beta+\gamma+m+n+1)},
\end{aligned}
$$




$$
c_{n}=\frac{(n+1)(\gamma+m+1)}{\beta+\gamma+m+n+2}-\frac{n(\gamma+m)}{\beta+\gamma+m+n} .
$$

It must be noted that (4.2) is essentially the three term recurrence relation for Jacobi polynomials [31].

It is clear that

$$
A_{n} \delta_{x}^{2} \phi_{n}(x, \alpha)+B_{n} \delta_{x} \phi_{n}(x, \alpha)+C_{n} \phi_{n}(x, \alpha)=0
$$

where

$$
A_{n}=1-x, \quad B_{n}=\alpha+\gamma-x(\alpha+\beta+\gamma+1), \quad C_{n}=x n(\alpha+\beta+\gamma+n+1) .
$$

Then for $m \geq n, M_{m, n}^{(\beta, \gamma)}\left(z_{1}, z_{2}\right)$ satisfy the following second-order partial differential equation

$$
\begin{aligned}
& \left(1-z_{1} z_{2}\right) \delta_{z_{2}}^{2} f\left(z_{1}, z_{2}\right)+\left\{m-n+\gamma-z_{1} z_{2}(\beta+\gamma+m-n+1)\right\} \delta_{z_{2}} f\left(z_{1}, z_{2}\right) \\
& \quad+z_{1} z_{2} n(\beta+\gamma+m+1) f\left(z_{1}, z_{2}\right)=0 .
\end{aligned}
$$

An equivalent form is

$$
\begin{aligned}
& \left(1-z_{1} z_{2}\right) z_{2} \frac{\partial^{2} f\left(z_{1}, z_{2}\right)}{\partial z_{2}^{2}}+\left[1+m-n+\gamma-z_{1} z_{2}(2+\gamma+\beta+m-n)\right] \frac{\partial f\left(z_{1}, z_{2}\right)}{\partial z_{2}} \\
& \quad+z_{1} n(m+\beta+\gamma+1) f\left(z_{1}, z_{2}\right)=0 .
\end{aligned}
$$

Similarly we establish

$$
\begin{aligned}
& \left(1-z_{1} z_{2}\right) z_{1} \frac{\partial^{2} f\left(z_{1}, z_{1}\right)}{\partial z_{2}^{1}}+\left[1-m+n+\gamma-z_{1} z_{2}(2+\gamma+\beta-m+n)\right] \frac{\partial f\left(z_{1}, z_{2}\right)}{\partial z_{2}} \\
& \quad+z_{2} m(n+\beta+\gamma+1) f\left(z_{1}, z_{2}\right)=0 .
\end{aligned}
$$

Theorem 4.1. For $m \geq n \geq 0, \beta>-1, \gamma>-1$ we have

$$
\begin{aligned}
& \frac{\partial}{\partial z_{2}} M_{m, n}^{(\beta, \gamma)}\left(z_{1}, z_{2}\right)=-(\beta+\gamma+m+1) M_{m, n}^{(\beta+1, \gamma)}\left(z_{1}, z_{2}\right), \\
& \delta_{z_{2}} M_{m, n}^{(\beta, \gamma)}\left(z_{1}, z_{2}\right)=n M_{m, n}^{(\beta, \gamma)}\left(z_{1}, z_{2}\right)-(\gamma+m) M_{m-1, n-1}^{(\beta+1, \gamma)}\left(z_{1}, z_{2}\right) \\
& =(\beta+\gamma+m+1)\left(M_{m, n}^{(\beta+1, \gamma)}\left(z_{1}, z_{2}\right)-M_{m, n}^{(\beta, \gamma)}\left(z_{1}, z_{2}\right)\right), \\
& \delta_{z_{1}} M_{m, n}^{(\beta, \gamma)}\left(z_{1}, z_{2}\right)=m M_{m, n}^{(\beta, \gamma)}\left(z_{1}, z_{2}\right)-(\gamma+m) M_{m-1, n-1}^{(\beta+1, \gamma)}\left(z_{1}, z_{2}\right) \\
& =(\beta+\gamma+m+1) M_{m, n}^{(\beta+1, \gamma)}\left(z_{1}, z_{2}\right)-(\beta+\gamma+n+1) M_{m, n}^{(\beta, \gamma)}\left(z_{1}, z_{2}\right), \\
& (\beta+\gamma+m+1) \delta_{z_{1}} M_{m, n}^{(\beta, \gamma)}\left(z_{1}, z_{2}\right)-(\beta+\gamma+n+1) \delta_{z_{2}} M_{m, n}^{(\beta, \gamma)}\left(z_{1}, z_{2}\right) \\
& =(m-n)(\beta+\gamma+m+1) M_{m, n}^{(\beta+1, \gamma)}\left(z_{1}, z_{2}\right) \text {, } \\
& \left(\delta_{z_{1}}-\delta_{z_{2}}\right) M_{m, n}^{(\beta, \gamma)}\left(z_{1}, z_{2}\right)=(m-n) M_{m, n}^{(\beta, \gamma)}\left(z_{1}, z_{2}\right), \\
& (\beta+\gamma+m+1) \delta_{z_{1}} M_{m, n}^{(\beta, \gamma)}\left(z_{1}, z_{2}\right)-(\beta+\gamma+n+1) \delta_{z_{2}} M_{m, n}^{(\beta, \gamma)}\left(z_{1}, z_{2}\right) \\
& =(m-n)(\beta+\gamma+m+1) M_{m, n}^{(\beta+1, \gamma)}\left(z_{1}, z_{2}\right) \text {. }
\end{aligned}
$$

Proof. The first equation easily follows from the definition. From the definition for $M_{m, n}^{(\beta, \gamma)}\left(z_{1}, z_{2}\right)$ we get

$$
\delta_{z_{2}} M_{m, n}^{(\beta, \gamma)}\left(z_{1}, z_{2}\right)=\sum_{k=0}^{n} \frac{(\beta+\gamma+m+1)_{n-k}(\gamma+1)_{m} z_{1}^{m-k}\left(-z_{2}\right)^{n-k}(n-k)}{k !(n-k) !(\gamma+1)_{m-k}}
$$




$$
\begin{aligned}
= & \sum_{k=0}^{n} \frac{(\beta+\gamma+m+1)_{n-k+1}(\gamma+1)_{m} z_{1}^{m-k}\left(-z_{2}\right)^{n-k}}{k !(n-k) !(\gamma+1)_{m-k}} \\
& -(\beta+\gamma+m+1) \sum_{k=0}^{n} \frac{(\beta+\gamma+m+1)_{n-k}(\gamma+1)_{m} z_{1}^{m-k}\left(-z_{2}\right)^{n-k}}{k !(n-k) !(\gamma+1)_{m-k}} \\
= & (\beta+\gamma+m+1) M_{m, n}^{(\beta+1, \gamma)}\left(z_{1}, z_{2}\right)-(\beta+\gamma+m+1) M_{m, n}^{(\beta, \gamma)}\left(z_{1}, z_{2}\right)
\end{aligned}
$$

and

$$
\begin{aligned}
\delta_{z_{2}} M_{m, n}^{(\beta, \gamma)}\left(z_{1}, z_{2}\right)= & \sum_{k=0}^{n} \frac{(\beta+\gamma+m+1)_{n-k}(\gamma+1)_{m} z_{1}^{m-k}\left(-z_{2}\right)^{n-k}(n-k)}{k !(n-k) !(\gamma+1)_{m-k}} \\
= & n \sum_{k=0}^{n} \frac{(\beta+\gamma+m+1)_{n-k}(\gamma+1)_{m} z_{1}^{m-k}\left(-z_{2}\right)^{n-k}}{k !(n-k) !(\gamma+1)_{m-k}} \\
& -\sum_{k=1}^{n} \frac{(\beta+\gamma+m+1)_{n-k}(\gamma+1)_{m} z_{1}^{m-k}\left(-z_{2}\right)^{n-k}}{(k-1) !(n-k) !(\gamma+1)_{m-k}} \\
= & n M_{m, n}^{(\beta, \gamma)}\left(z_{1}, z_{2}\right)-(\gamma+m) M_{m-1, n-1}^{(\beta+1, \gamma)}\left(z_{1}, z_{2}\right) .
\end{aligned}
$$

Similarly,

$$
\begin{aligned}
\delta_{z_{1}} M_{m, n}^{(\beta, \gamma)}\left(z_{1}, z_{2}\right)= & \sum_{k=0}^{n} \frac{(\beta+\gamma+m+1)_{n-k}(\gamma+1)_{m} z_{1}^{m-k}\left(-z_{2}\right)^{n-k}(m-k)}{k !(n-k) !(\gamma+1)_{m-k}} \\
= & m \sum_{k=0}^{n} \frac{(\beta+\gamma+m+1)_{n-k}(\gamma+1)_{m} z_{1}^{m-k}\left(-z_{2}\right)^{n-k}}{k !(n-k) !(\gamma+1)_{m-k}} \\
& -\sum_{k=0}^{n-1} \frac{(\beta+\gamma+m+1)_{n-1-k}(\gamma+1)_{m} z_{1}^{m-1-k}\left(-z_{2}\right)^{n-1-k}}{k !(n-1-k) !(\gamma+1)_{m-1-k}} \\
= & m M_{m, n}^{(\beta, \gamma)}\left(z_{1}, z_{2}\right)-(\gamma+m) M_{m-1, n-1}^{(\beta+1, \gamma)}\left(z_{1}, z_{2}\right), \\
\delta_{z_{1}} M_{m, n}^{(\beta, \gamma)}\left(z_{1}, z_{2}\right)= & \sum_{k=0}^{n} \frac{(\beta+\gamma+m+1)_{n-k+1}(\gamma+1)_{m} z_{1}^{m-k}\left(-z_{2}\right)^{n-k}}{k !(n-k) !(\gamma+1)_{m-k}}-(\beta+\gamma+n+1) \\
= & \sum_{k=0}^{n} \frac{(\beta+\gamma+m+1)_{n-k}(\gamma+1)_{m} z_{1}^{m-k}\left(-z_{2}\right)^{n-k}}{k !(n-k) !(\gamma+1)_{m-k}} \\
= & (\beta+\gamma+m+1) M_{m, n}^{(\beta+1, \gamma)}\left(z_{1}, z_{2}\right)-(\beta+\gamma+n+1) M_{m, n}^{(\beta, \gamma)}\left(z_{1}, z_{2}\right) .
\end{aligned}
$$

Ismail and Zeng [20] used the generating function

$$
\sum_{n=0}^{\infty} P_{n}^{(\alpha, \beta)}(1-2 x) z^{n}=\frac{2^{\alpha+\beta}}{R(1+z+R)^{\beta}(1-z+R)^{\alpha}}
$$

where

$$
R=\sqrt{(1-z)^{2}+4 x z},
$$

[28, Section 140] to establish the following generating functions:

$$
\sum_{m, n=0}^{\infty} M_{m, n}^{(\beta, \gamma)}\left(z_{1}, z_{2}\right) u^{m} v^{n}=\frac{2^{\beta+\gamma}}{\rho}(1+u v+\rho)^{-\beta}(1-u v+\rho)^{-\gamma}
$$




$$
\times\left[\frac{1}{1-2 z_{1} u /(1+\rho-u v)}+\frac{1}{1-2 v z_{2} /(1+\rho-u v)}-1\right],
$$

and

$$
\sum_{m \geq n \geq 0} M_{m, n}^{(\beta, \gamma)}\left(z_{1}, z_{2}\right) \frac{u^{m} v^{n}}{(m-n) !}=\frac{2^{\beta+\gamma}}{\rho}(1+u v+\rho)^{-\beta}(1-u v+\rho)^{-\gamma} \exp \left(\frac{2 z_{1} u}{1-u v+\rho}\right),
$$

where

$$
\rho=\left(1-2 u v\left(1-2 z_{1} z_{2}\right)+u^{2} v^{2}\right)^{1 / 2}
$$

They also verified the limiting relation

$$
\lim _{\beta \rightarrow \infty} M_{m, n}^{(\beta, \gamma)}\left(z_{1}, z_{2} / \beta\right)=z_{1}^{m-n} L_{n}^{(\gamma+m-n)}\left(z_{1} z_{2}\right)=(-1)^{n} Z_{m, n}^{(\gamma)}\left(z_{1}, z_{2}\right) .
$$

One can use the generating function (4.3) to establish the generating relation

$$
\sum_{m \geq n \geq 0} u^{m} v^{n} M_{m, n}^{(\beta, \gamma)}\left(z_{1}, z_{2}\right)=\frac{2^{\beta+\gamma}(1+u v+\rho)^{-\beta}(1-u v+\rho)^{1-\gamma}}{\rho\left(1-u v-2 u z_{1}+\rho\right)}
$$

with $\rho$ as in (4.4).

\section{The polynomials $\left\{Z_{m, n}^{(\beta)}\left(z_{1}, z_{2} \mid q\right)\right\}$}

For $\alpha>-1$, the moment problem associated with $q$-Laguerre polynomials

$$
L_{n}^{(\alpha)}(x ; q)=\left(q^{\alpha+1} ; q\right)_{n} \sum_{k=0}^{n} \frac{q^{(\alpha+n-k)(n-k)}(-x)^{n-k}}{(q ; q)_{k}\left(q, q^{\alpha+1} ; q\right)_{n-k}} .
$$

is indeterminate. It is well-known that

$$
\int_{0}^{\infty} L_{m}^{(\alpha)}(x ; q) L_{n}^{(\alpha)}(x ; q) x^{\alpha} d \mu(x \mid q)=\zeta_{n}(\alpha) \delta_{m, n},
$$

where $\mu(x \mid q), \zeta_{n}(\alpha)$ are given by

$$
\begin{aligned}
& d \mu(x \mid q)=\frac{x^{\alpha} d x}{(-x ; q)_{\infty}}, \\
& \zeta_{n}(\alpha)=\Gamma(-\alpha) \Gamma(\alpha+1) \frac{\left(q^{-\alpha} ; q\right)_{\infty}}{(q ; q)_{\infty}} \frac{\left(q^{\alpha+1} ; q\right)_{n}}{q^{n}(q ; q)_{n}} \\
& d \mu(x \mid q)=\sum_{k=-\infty}^{\infty} \frac{x \delta\left(x-c q^{k}\right)}{(-x ; q)_{\infty}} \\
& \zeta_{n}(\alpha)=\frac{\left(q,-c q^{\alpha+1},-\frac{q^{-\alpha}}{c} ; q\right)_{\infty}}{\left(q^{\alpha+1},-c,-\frac{q}{c} ; q\right)_{\infty} c^{-\alpha-1}} \frac{\left(q^{\alpha+1} ; q\right)_{n}}{(q ; q)_{n} q^{n}}
\end{aligned}
$$

and

$$
\begin{aligned}
& d \mu(x \mid q)=\frac{x^{-c}\left(-\lambda x,-\frac{q}{\lambda x} ; q\right)_{\infty} d x}{\left(-x,-\lambda q^{c} x,-\frac{q}{\lambda q^{c} x} ; q\right)_{\infty}} \\
& \zeta_{n}(\alpha)=\delta_{m, n} \int_{0}^{\infty} \frac{x^{\alpha-c}\left(-\lambda x,-\frac{q}{\lambda x} ; q\right)_{\infty} d x}{\left(-x,-\lambda q^{c} x,-\frac{q}{\lambda q^{c} x} ; q\right)_{\infty}}, \quad c, \lambda>0
\end{aligned}
$$


where $c, \lambda>0$. Following the procedure outline in Section 2 we let

$$
z_{m, n}^{(\beta)}\left(z_{1}, z_{2} \mid q\right)= \begin{cases}z_{1}^{m-n} L_{n}^{(\beta+m-n)}\left(z_{1} z_{2} ; q\right), & m \geq n, \\ z_{n, m}^{(\beta)}\left(z_{2}, z_{1} \mid q\right), & m<n,\end{cases}
$$

then we have

$$
\begin{aligned}
& z_{m, n}^{(\beta)}\left(z_{1}, z_{2} \mid q\right)=\sum_{k=0}^{n} \frac{q^{(\beta+m-k)(n-k)}\left(q^{\beta+1} ; q\right)_{m} z_{1}^{m-k} z_{2}^{n-k}}{(-1)^{n-k}(q ; q)_{k}(q ; q)_{n-k}\left(q^{\beta+1} ; q\right)_{m-k}} \\
& z_{1} z_{m, n}^{(\beta+1)}\left(z_{1}, z_{2} \mid \beta\right)=z_{m+1, n}^{(\beta)}\left(z_{1}, z_{2} \mid \beta\right)
\end{aligned}
$$

and

$$
z_{m, n}^{(0)}\left(z_{1}, z_{2} \mid q\right)=\frac{(-1)^{n}}{(q ; q)_{n}} h_{m, n}\left(z_{1}, z_{2} \mid q\right) .
$$

for $m \geq n$. Applying Theorem 2.1 we obtain the following result

Theorem 5.1. For $m, n, s, t=0,1, \ldots$ and $\beta>-1$ we have the following orthogonality relations

$$
\int_{\mathbb{R}^{2}} z_{m, n}^{(\beta)}(z, \bar{z} \mid q) \overline{z_{s, t}^{(\beta)}(z, \bar{z} \mid q)} r^{2 \beta} d \mu\left(r^{2} \mid q\right) d \theta=\zeta_{m \wedge n}(|m-n|+\beta) \delta_{m, s} \delta_{n, t},
$$

where $d \mu(x \mid q), \zeta_{n}(\alpha)$ may be any pairs (5.1), (5.2) or (5.3).

Applying formulas (2.5) and (2.7) in the case

$$
c_{k}(n, \alpha)=\frac{\left(q^{\alpha+1} ; q\right)_{n} q^{(\alpha+n-k)(n-k)}(-1)^{n-k}}{(q ; q)_{k}\left(q, q^{\alpha+1} ; q\right)_{n-k}}
$$

we obtain the recurrences stated as a theorem below.

Theorem 5.2. For $\beta>-1$ and $m \geq n$ we have

$$
\begin{aligned}
& q^{(m+1+\beta)} z_{2} z_{m+1, n}^{(\beta)}\left(z_{1}, z_{2}\right)=-\left(1-q^{n+1}\right) z_{m+1, n+1}^{(\beta)}\left(z_{1}, z_{2}\right)+\left(1-q^{m+\beta+1}\right) z_{m, n}^{(\beta)}\left(z_{1}, z_{2}\right), \\
& q^{n} z_{1} z_{m, n+1}^{(\beta)}\left(z_{1}, z_{2}\right)=z_{m+1, n}^{(\beta)}\left(z_{1}, z_{2}\right)-z_{m, n-1}^{(\beta)}\left(z_{1}, z_{2}\right)
\end{aligned}
$$

and

$$
\begin{aligned}
& \left(1+q\left(1-q^{n}-q^{m+\beta}\right)-z_{1} z_{2} q^{\beta+m+n+1}\right) z_{m, n}^{(\beta)}\left(z_{1}, z_{2}\right) \\
& \quad=\left(1-q^{1+n}\right) z_{m+1, n+1}^{(\beta)}\left(z_{1}, z_{2}\right)+q\left(1-q^{\beta+m}\right) z_{m-1, n-1}^{(\beta)}\left(z_{1}, z_{2}\right) .
\end{aligned}
$$

The $q$-Laguerre polynomials satisfy the following $q$-difference operators

$$
\begin{aligned}
& D_{q, x} L_{n}^{(\alpha)}(x ; q)=-\frac{q^{\alpha+1}}{1-q} L_{n-1}^{(\alpha+1)}(q z ; q), \\
& D_{q, x}\left\{w_{\alpha}(x ; q) L_{n}^{(\alpha)}(x ; q)\right\}=\frac{1-q^{n+1}}{1-q} w_{\alpha-1}(x ; q) L_{n+1}^{(\alpha-1)}(z ; q), \\
& w_{\alpha}(x ; q) L_{n}^{(\alpha)}(x ; q)=\frac{(1-q)^{n}}{(q ; q)_{n}} D_{q, x}^{n}\left\{w_{\alpha+n}(x ; q)\right\},
\end{aligned}
$$

and

$$
w_{\beta}(x ; q)=\frac{x^{\beta}}{(-x ; q)_{\infty}} .
$$


Theorem 5.3. For $\beta>-1$ we have

$$
\begin{aligned}
& \frac{D_{q, z_{1}}\left(z_{1}^{n-m} z_{m, n}^{(\beta)}\left(z_{1}, z_{2}\right)\right)}{z_{1}^{n-m}}=\frac{q^{\beta}}{q-1} \frac{z_{2}}{z_{1}} z_{m, n-1}^{(\beta)}\left(q z_{1}, z_{2}\right), \\
& D_{q, z_{2}}\left(z_{m, n}^{(\beta)}\left(z_{1}, z_{2}\right)\right)=\frac{q^{\beta}}{q-1} z_{m, n-1}^{(\beta)}\left(q z_{1}, z_{2}\right), \\
& z_{m, n}^{(\beta)}\left(z_{1}, z_{2}\right)=\frac{(1-q)^{m+n} D_{q, z_{1}}^{n}\left\{z_{2}^{\beta} D_{q, z_{2}}^{m}\left\{z_{2}^{-\beta} w_{\beta}\left(z_{1} z_{2}\right)\right\}\right\}}{(-1)^{m}(q ; q)_{n} w_{\beta}\left(z_{1} z_{2}\right)} \\
& \frac{z_{2} D_{q, z_{2}}\left\{w_{\beta}\left(z_{1} z_{2}\right) z_{m, n}^{(\beta)}\left(z_{1}, z_{2}\right)\right\}}{w_{\beta}\left(z_{1} z_{2}\right)}=\frac{1-q^{\beta}}{1-q} z_{m, n}^{(\beta)}\left(z_{1}, z_{2}\right)-\frac{q^{\beta} z_{2}}{1-q} z_{m+1, n}^{(\beta)}\left(z_{1}, z_{2}\right)
\end{aligned}
$$

for $m \geq n$ and

$$
\begin{aligned}
& \frac{D_{q, z_{1}}\left\{w_{\beta}\left(z_{1} z_{2} ; q\right) z_{m, n}^{(\beta)}\left(z_{1}, z_{2}\right)\right\}}{w_{\beta}\left(z_{1} z_{2} ; q\right)}=\frac{1-q^{n+1}}{1-q} z_{m, n+1}^{(\beta)}\left(z_{1}, z_{2}\right), \\
& \frac{D_{q, z_{2}}\left\{z_{2}^{m-n} w_{\beta}\left(z_{1} z_{2} ; q\right) z_{m, n}^{(\beta)}\left(z_{1}, z_{2}\right)\right\}}{z_{2}^{m-n} w_{\beta}\left(z_{1} z_{2} ; q\right)}=\frac{1-q^{n+1}}{1-q} \frac{z_{1}}{z_{2}} z_{m, n+1}^{(\beta)}\left(z_{1}, z_{2}\right)
\end{aligned}
$$

for $m>n$.

Proof. First we observe that

$$
D_{q, x}\left\{x^{-\beta} w_{\beta}(x)\right\}=\frac{1}{q-1} x^{-\beta} w_{\beta}(x)
$$

to get

$$
z_{2}^{\beta+m} D_{q, z_{2}}^{m}\left\{z_{2}^{-\beta} w_{\beta}\left(z_{1} z_{2}\right)\right\}=\frac{w_{\beta+m}\left(z_{1} z_{2}\right)}{(q-1)^{m}}
$$

and

$$
D_{q, z_{1}}^{n}\left\{z_{2}^{\beta+m} D_{q, z_{2}}^{m}\left\{z_{2}^{-\beta} w_{\beta}\left(z_{1} z_{2}\right)\right\}\right\}=\frac{D_{q, z_{1}}^{n}\left\{w_{\beta+m}\left(z_{1} z_{2}\right)\right\}}{(q-1)^{m}}=\frac{z_{2}^{n}}{(q-1)^{m}} D_{q, z_{1} z_{2}}^{n}\left\{w_{\beta+m}\left(z_{1} z_{2}\right)\right\},
$$

or

$$
\begin{aligned}
D_{q, z_{1}}^{n} & \left\{z_{2}^{\beta+m-n} D_{q, z_{2}}^{m}\left\{z_{2}^{-\beta} w_{\beta}\left(z_{1} z_{2}\right)\right\}\right\} \\
& =\frac{D_{q, z_{1} z_{2}}^{n}\left\{w_{\beta+m}\left(z_{1} z_{2}\right)\right\}}{(q-1)^{m}}=\frac{(-1)^{m}(q ; q)_{n}}{(1-q)^{m+n}} z_{2}^{m-n} z_{m, n}^{(\beta)}\left(z_{1}, z_{2}\right),
\end{aligned}
$$

which is (5.9), (5.7) and (5.8) are obtained from (5.5), and (5.11) and (5.12) are derived from (5.6).

From (5.9) we get

$$
\begin{aligned}
D_{q, z_{2}}\left\{w_{\beta}\left(z_{1} z_{2}\right) z_{m, n}^{(\beta)}\left(z_{1}, z_{2}\right)\right\}=\frac{(1-q)^{m+n}}{(-1)^{m}(q ; q)_{n}} D_{q, z_{1}}^{n}\left\{D_{q, z_{2}}\left\{z_{2}^{\beta} D_{q, z_{2}}^{m}\left\{z_{2}^{-\beta} w_{\beta}\left(z_{1} z_{2}\right)\right\}\right\}\right\} \\
=\frac{(1-q)^{m+n-1}\left(1-q^{\beta}\right)}{(-1)^{m}(q ; q)_{n}} D_{q, z_{1}}^{n}\left\{z_{2}^{\beta-1} D_{q, z_{2}}^{m}\left\{z_{2}^{-\beta} w_{\beta}\left(z_{1} z_{2}\right)\right\}\right\} \\
\quad+\frac{(1-q)^{m+n} q^{\beta}}{(-1)^{m}(q ; q)_{n}} D_{q, z_{1}}^{n}\left\{z_{2}^{\beta} D_{q, z_{2}}^{m+1}\left\{z_{2}^{-\beta} w_{\beta}\left(z_{1} z_{2}\right)\right\}\right\}
\end{aligned}
$$

which gives (5.10). 
The $q$-Laguerre polynomials satisfy the following second-order difference equation

$$
-q^{\alpha}\left(1-q^{n}\right) x y(x)=q^{\alpha}(1+x) y(q x)-\left(1+q^{\alpha}(1+x)\right) y(x)+y\left(q^{-1} x\right)
$$

or

$$
(1+q x) \theta_{q, x}^{2} y(x)+\frac{1-q^{\alpha}-2 q^{\alpha+1} x+q^{\alpha+n+1} x}{q^{\alpha}(1-q)} \theta_{q, x} y(x)+\frac{q x\left(1-q^{n}\right)}{(1-q)^{2}} y(x)=0 .
$$

where $y(x)=L_{n}^{(\alpha)}(x ; q), \theta_{q, z}=z D_{q, z}$.

Theorem 5.4. For $m \geq n$ the function $f=z_{m, n}^{(\beta)}\left(z_{1}, z_{2}\right)$ satisfies the second-order $q$-difference equations

$$
\left(1+q z_{1} z_{2}\right) \theta_{q, z_{2}}^{2} f+\frac{q^{n-m-\beta}-1-\left(2-q^{n}\right) q z_{1} z_{2}}{1-q} \theta_{q, z_{2}} f+\frac{q z_{1} z_{2}\left(1-q^{n}\right)}{(1-q)^{2}} f=0
$$

and

$$
\left(1+q z_{1} z_{2}\right) \theta_{q, z_{1}}^{2} f-\frac{1-q^{\beta+m-n}+\left(2-q^{\beta+m+1}\right) q z_{1} z_{2}}{1-q} \theta_{q, z_{1}} f+\frac{q\left(1-q^{\beta+m}\right) z_{1} z_{2}}{(1-q)^{2}} f=0 .
$$

Starting with the little $q$-Laguerre [23] or Wall polynomials [5]

$$
p_{n}\left(x ; q^{\alpha} \mid q\right)=\sum_{k=0}^{n} \frac{(q ; q)_{n} q^{\frac{(k-n)(n+k-1)}{2}}(-x)^{n-k}}{(q ; q)_{k}\left(q, q^{\alpha+1} ; q\right)_{n-k}}
$$

we define the $2 \mathrm{D}$ polynomials $w_{m, n}^{(\beta)}\left(z_{1}, z_{2} \mid q\right)$ through

$$
w_{m, n}^{(\beta)}\left(z_{1}, z_{2} \mid q\right)= \begin{cases}z_{1}^{m-n} p_{n}\left(z_{1} z_{2} ; q^{\beta+m-n} \mid q\right), & m \geq n, \\ w_{n, m}^{(\beta)}\left(z_{2}, z_{1} \mid q\right), & m<n,\end{cases}
$$

for $\beta>-1$ and $m, n=0,1, \ldots$ Then for $m \geq n$ find that

$$
\begin{aligned}
& z_{1} w_{m, n}^{(\beta+1)}\left(z_{1}, z_{2} \mid \beta\right)=w_{m+1, n}^{(\beta)}\left(z_{1}, z_{2} \mid \beta\right) \\
& w_{m, n}^{(\beta)}\left(z_{1}, z_{2} \mid q\right)=\sum_{k=0}^{n} \frac{(q ; q)_{n}\left(q^{\beta+1} ; q\right)_{m-n} z_{1}^{m-k} z_{2}^{n-k} q^{-\left(\begin{array}{c}
k \\
2
\end{array}\right)-\left(\begin{array}{c}
n \\
2
\end{array}\right)}}{(-1)^{n-k}(q ; q)_{k}(q ; q)_{n-k}\left(q^{\beta+1} ; q\right)_{m-k}} .
\end{aligned}
$$

The orthogonality relation for the little $q$-Laguerre polynomials is

$$
\begin{aligned}
& \sum_{k=0}^{\infty} q^{(\alpha+1) k}\left(q^{k+1} ; q\right)_{\infty} p_{m}\left(q^{k} ; q^{\alpha} \mid q\right) p_{n}\left(q^{k} ; q^{\alpha} \mid q\right) \\
& \quad=\frac{(q ; q)_{\infty} q^{(\alpha+1) n}(q ; q)_{n} \delta_{m, n}}{\left(q^{\alpha+1} ; q\right)_{\infty}\left(q^{\alpha+1} ; q\right)_{n}}, \quad m, n=0,1, \ldots
\end{aligned}
$$

This leads to the orthogonality relation $\left\{w_{m, n}^{(\beta)}\left(z_{1}, z_{2} \mid q\right)\right\}_{m, n=0}^{\infty}$ in the form

Theorem 5.5. For $m, n, s, t=0,1, \ldots$ and $\beta>-1$ we have the following orthogonality relations

$$
\int_{\mathbb{R}^{2}} w_{m, n}^{(\beta)}(z, \bar{z} \mid q) \overline{w_{s, t}^{(\beta)}(z, \bar{z} \mid q)} r^{2 \beta} d \mu\left(r^{2} \mid q\right) d \theta=\zeta_{m \wedge n}(|m-n|+\beta) \delta_{m, s} \delta_{n, t}
$$


where $z=r e^{i \theta}$

$$
d \mu(r \mid q)=\sum_{k=0}^{\infty} x(x q ; q)_{\infty} \delta\left(x-q^{k}\right)
$$

and

$$
\zeta_{n}(\alpha)=\frac{(q ; q)_{\infty} q^{(\alpha+1) n}(q ; q)_{n}}{\left(q^{\alpha+1} ; q\right)_{\infty}\left(q^{\alpha+1} ; q\right)_{n}}
$$

The function $y(x)=p_{n}\left(x ; q^{\alpha} \mid q\right)$ satisfies the $q$-difference equation

$$
q^{\alpha} y\left(q^{2} z\right)-\left(1+q^{\alpha}-z q^{1-n}\right) y(q z)+(1-q z) y(z)=0
$$

or equivalently

$$
q^{\alpha+n-1} \theta_{q, z}^{2} y(z)+\frac{q^{n-1}+q^{\alpha+n-1}-z}{1-q} \theta_{q, z} y(z)+\frac{z\left(1-q^{n}\right)}{(1-q)^{2}} y(z)=0,
$$

where $\theta_{q, z}=z D_{q, z}$. This leads to the following theorem.

Theorem 5.6. For $\beta>-1, m \geq n$ the polynomial $w_{m, n}^{(\beta)}\left(z_{1}, z_{2} \mid q\right)$, satisfies the $q$-partial difference equations

$$
q^{\beta+m-1} \theta_{q, z_{2}}^{2} f+\frac{q^{n-1}-q^{\beta+m-1}-z_{1} z_{2}}{1-q} \theta_{q, z_{2}} f+\frac{\left(1-q^{n}\right) z_{1} z_{2}}{(1-q)^{2}} f=0,
$$

and

$$
q^{n} \theta_{q, z_{1}}^{2} f-\frac{q^{n}-q^{\beta+m}+q z_{1} z_{2}}{1-q} \theta_{q, z_{1}} f+\frac{q\left(1-q^{\beta+m}\right) z_{1} z_{2}}{(1-q)^{2}} f=0 .
$$

From

$$
\begin{aligned}
& D_{q, x} p_{n}\left(x ; q^{\alpha} \mid q\right)=-\frac{q^{1-n}\left(1-q^{n}\right)}{(1-q)\left(1-q^{\alpha+1}\right)} p_{n-1}\left(x ; q^{\alpha+1} \mid q\right), \\
& \frac{D_{q^{-1}, x}\left\{w\left(x ; q^{\alpha}\right) p_{n}\left(x ; q^{\alpha} \mid q\right)\right\}}{w(x ; \alpha-1)}=\frac{\left(1-q^{\alpha}\right) p_{n+1}\left(x ; q^{\alpha-1} \mid q\right)}{q^{\alpha-1}(1-q)}
\end{aligned}
$$

and

$$
w(x ; \alpha \mid q) p_{n}\left(x ; q^{\alpha} \mid q\right)=\frac{q^{n \alpha+\left(\begin{array}{c}
n \\
2
\end{array}\right)}(1-q)^{n}}{\left(q^{\alpha+1} ; q\right)_{n}} D_{q^{-1}, x}^{n} w(x ; \alpha+n \mid q),
$$

where

$$
w(x ; \alpha)=(q x ; q)_{\infty} x^{\alpha}
$$

we get the following relations:

Theorem 5.7. For $\beta>-1$ and $m \geq n$ we have

$$
\begin{aligned}
& \frac{D_{q, z_{1}}\left\{z_{1}^{n-m} w_{m, n}^{(\beta)}\left(z_{1}, z_{2} \mid q\right)\right\}}{z_{1}^{n-m}}=-\frac{z_{2}}{z_{1}} \frac{q^{1-n}\left(1-q^{n}\right) w_{m, n-1}^{(\beta)}\left(z_{1}, z_{2} \mid q\right)}{(1-q)\left(1-q^{\beta+m-n+1}\right)}, \\
& D_{q, z_{2}}\left\{w_{m, n}^{(\beta)}\left(z_{1}, z_{2} \mid q\right)\right\}=-\frac{q^{1-n}\left(1-q^{n}\right) w_{m, n-1}^{(\beta)}\left(z_{1}, z_{2} \mid q\right)}{(1-q)\left(1-q^{\beta+m-n+1}\right)}
\end{aligned}
$$


and

$$
\begin{aligned}
w\left(z_{1} z_{2} ; \beta \mid q\right) w_{m, n}^{(\beta)}\left(z_{1}, z_{2} \mid q\right)= & \frac{q^{m(n-1)+n(\beta-1)-\left(\begin{array}{c}
n \\
2
\end{array}\right)}(1-q)^{m+n}}{(-1)^{m}\left(q^{\beta+m-n+1} ; q\right)_{n}} \\
& \times D_{q^{-1}, z_{1}}^{n}\left\{\left(z_{1} z_{2}\right)^{\beta} D_{q^{-1}, z_{2}}^{m}\left\{\left(z_{1} z_{2}\right)^{-\beta} w\left(z_{1} z_{2} ; \beta \mid q\right)\right\}\right\} .
\end{aligned}
$$

For $m>n$ we have

$$
\begin{aligned}
& \frac{D_{q^{-1}, z_{1}}\left\{w\left(z_{1} z_{2} ; \beta \mid q\right) w_{m, n}^{(\beta)}\left(z_{1}, z_{2} \mid q\right)\right\}}{w\left(z_{1} z_{2} ; \beta \mid q\right)}=\frac{\left(1-q^{\beta+m-n}\right) w_{m, n+1}^{(\beta)}\left(z_{1}, z_{2} \mid q\right)}{q^{\beta+m-n-1}(1-q)}, \\
& \frac{D_{q^{-1}, z_{2}}\left\{z_{2}^{m-n} w\left(z_{1} z_{2} ; \beta \mid q\right) w_{m, n}^{(\beta)}\left(z_{1}, z_{2} \mid q\right)\right\}}{z_{2}^{m-n} w\left(z_{1} z_{2} ; \beta \mid q\right)}=\frac{z_{1}}{z_{2}} \frac{\left(1-q^{\beta+m-n}\right) w_{m, n+1}^{(\beta)}\left(z_{1}, z_{2} \mid q\right)}{q^{\beta+m-n-1}(1-q)} \\
& \frac{z_{2} D_{q^{-1}, z_{2}}\left\{w\left(z_{1} z_{2} ; \beta \mid q\right) w_{m, n}^{(\beta)}\left(z_{1}, z_{2} \mid q\right)\right\}}{w_{m, n}^{(\beta)}\left(z_{1}, z_{2} \mid q\right)} \\
& =\frac{1-q^{\beta}}{1-q} w_{m, n}^{(\beta)}\left(z_{1}, z_{2} \mid q\right)-\frac{q^{\beta+1-n}}{1-q} \frac{1-q^{\beta+m+1}}{1-q^{\beta+m-n+1}} z_{2} w_{m+1, n}^{(\beta)}\left(z_{1}, z_{2} \mid q\right)
\end{aligned}
$$

Proof. Observe that

$$
D_{q^{-1}, z_{1}}^{n}=z_{2}^{n} D_{q^{-1}, z_{1} z_{2}}^{n}
$$

and

$$
z_{1}^{m} w\left(z_{1} z_{2} ; \beta \mid q\right)=\left(1-q^{-1}\right)^{m}\left(z_{1} z_{2}\right)^{\beta} D_{q^{-1}, z_{2}}^{m}\left\{\left(z_{1} z_{2}\right)^{-\beta} w\left(z_{1} z_{2} ; \beta \mid q\right)\right\},
$$

then

$$
\begin{aligned}
w\left(z_{1} z_{2} ; \beta \mid q\right) w_{m, n}^{(\beta)}\left(z_{1}, z_{2} \mid q\right)= & \frac{q^{n(\beta+m)-\left(\begin{array}{c}
n+1 \\
2
\end{array}\right)}(1-q)^{n}}{\left(q^{\beta+m-n+1} ; q\right)_{n}} D_{q^{-1}, z_{1}}^{n}\left(z_{1}^{m} w\left(z_{1} z_{2} ; \beta \mid q\right)\right) \\
= & \frac{q^{m(n-1)+n(\beta-1)-\left(\begin{array}{c}
n \\
2
\end{array}\right)}(1-q)^{m+n}}{(-1)^{m}\left(q^{\beta+m-n+1} ; q\right)_{n}} \\
& \times D_{q^{-1}, z_{1}}^{n}\left\{\left(z_{1} z_{2}\right)^{\beta} D_{q^{-1}, z_{2}}^{m}\left\{\left(z_{1} z_{2}\right)^{-\beta} w\left(z_{1} z_{2} ; \beta \mid q\right)\right\}\right\}, \\
w\left(z_{1} z_{2} ; \beta \mid q\right) w_{m, n}^{(\beta)}\left(z_{1}, z_{2} \mid q\right)= & \frac{q^{m(n-1)+n(\beta-1)-\left(\begin{array}{c}
n \\
2
\end{array}\right)(1-q)^{m+n}}}{(-1)^{m}\left(q^{\beta+m-n+1} ; q\right)_{n}} \\
& \times D_{q^{-1}, z_{1}}^{n}\left\{\left(z_{1} z_{2}\right)^{\beta} D_{q^{-1}, z_{2}}^{m}\left\{\left(z_{1} z_{2}\right)^{-\beta} w\left(z_{1} z_{2} ; \beta \mid q\right)\right\}\right\},
\end{aligned}
$$

which gives (5.14), (5.15) and (5.16) are obtained from (5.13) by direct computation.

From (5.14) we have

$$
\begin{aligned}
D_{q^{-1}, z_{2}} & \left\{w\left(z_{1} z_{2} ; \beta \mid q\right) w_{m, n}^{(\beta)}\left(z_{1}, z_{2} \mid q\right)\right\}=\frac{q^{m(n-1)+n(\beta-1)-\left(\begin{array}{c}
n \\
2
\end{array}\right)(1-q)^{m+n}}}{(-1)^{m}\left(q^{\beta+m-n+1} ; q\right)_{n}} \\
& \times D_{q^{-1}, z_{1}}^{n}\left\{D_{q^{-1}, z_{2}}\left\{\left(z_{1} z_{2}\right)^{\beta} D_{q^{-1}, z_{2}}^{m}\left\{\left(z_{1} z_{2}\right)^{-\beta} w\left(z_{1} z_{2} ; \beta \mid q\right)\right\}\right\}\right\} \\
= & \frac{q^{m(n-1)+n(\beta-1)-\left(\begin{array}{c}
n \\
2
\end{array}\right)(1-q)^{m+n}}}{(-1)^{m}\left(q^{\beta+m-n+1} ; q\right)_{n}} q^{\beta} D_{q^{-1}, z_{1}}^{n}\left\{\left(z_{1} z_{2}\right)^{\beta} D_{q^{-1}, z_{2}}^{m+1}\left\{\left(z_{1} z_{2}\right)^{-\beta} w\left(z_{1} z_{2} ; \beta \mid q\right)\right\}\right\} \\
& +\frac{q^{m(n-1)+n(\beta-1)+1-\beta-\left(\begin{array}{c}
n \\
2
\end{array}\right)}(1-q)^{m+n-1}\left(1-q^{\beta}\right)}{(-1)^{m}\left(q^{\beta+m-n+1} ; q\right)_{n}} \\
& \times z_{2}^{-1} D_{q^{-1}, z_{1}}^{n}\left\{\left(z_{1} z_{2}\right)^{\beta} D_{q^{-1}, z_{2}}^{m}\left\{\left(z_{1} z_{2}\right)^{-\beta} w\left(z_{1} z_{2} ; \beta \mid q\right)\right\}\right\}
\end{aligned}
$$




$$
\begin{aligned}
= & -\frac{q^{\beta+1-n}}{1-q} \frac{1-q^{\beta+m+1}}{1-q^{\beta+m-n+1}} w\left(z_{1} z_{2} ; \beta \mid q\right) w_{m+1, n}^{(\beta)}\left(z_{1}, z_{2} \mid q\right) \\
& +\frac{\left(1-q^{\beta}\right)}{z_{2}(1-q)} w\left(z_{1} z_{2} ; \beta \mid q\right) w_{m, n}^{(\beta)}\left(z_{1}, z_{2} \mid q\right),
\end{aligned}
$$

which gives (5.17).

Applying (2.8)-(2.11) and (2.13) to

$$
c_{k}(n, \alpha)=\frac{(q ; q)_{n} q^{\frac{(k-n)(n+k-1)}{2}}(-1)^{n-k}}{(q ; q)_{k}\left(q, q^{\alpha+1} ; q\right)_{n-k}}
$$

we get the following recurrences:

Theorem 5.8. For $\beta>-1, m \geq n$ we have

$$
\begin{aligned}
& \frac{z_{2} w_{m+1, n}^{(\beta)}\left(z_{1}, z_{2} \mid q\right)}{q^{n}\left(1-q^{m-n+\beta+1}\right)}=w_{m, n}^{(\beta)}\left(z_{1}, z_{2} \mid q\right)-w_{m+1, n+1}^{(\beta)}\left(z_{1}, z_{2} \mid q\right), \\
& z_{1}\left(1-q^{m-n+\beta+1}\right) w_{m, n}^{(\beta)}\left(z_{1}, z_{2} \mid q\right) \\
& \quad \quad=\left(1-q^{m+\beta+1}\right) w_{m+1, n}^{(\beta)}\left(z_{1}, z_{2} \mid q\right)-q^{m-n+\beta+1}\left(1-q^{n}\right) w_{m, n-1}^{(\beta)}\left(z_{1}, z_{2} \mid q\right),
\end{aligned}
$$

and

$$
\begin{aligned}
& \left(q^{n}+q^{m+\beta}\left(1-q^{n}-q^{n+1}\right)-z_{1} z_{2}\right) w_{m, n}^{(\beta)}\left(z_{1}, z_{2} \mid q\right) \\
& \quad=q^{n}\left(1-q^{m+\beta+1}\right) w_{m+1, n+1}^{(\beta)}\left(z_{1}, z_{2} \mid q\right)+q^{m+\beta}\left(1-q^{n}\right) w_{m-1, n-1}^{(\beta)}\left(z_{1}, z_{2} \mid q\right) .
\end{aligned}
$$

\section{The polynomials $\left\{M_{n}^{(\alpha, \beta)}\left(z_{1}, z_{2} \mid q\right)\right\}$}

For $\alpha, \gamma>-1$, the Little $q$-Jacobi polynomials

$$
p_{n}\left(x ; q^{\alpha}, q^{\gamma} \mid q\right)=\frac{(q ; q)_{n}}{q^{\left(\begin{array}{c}
n \\
2
\end{array}\right)}} \sum_{k=0}^{n} \frac{\left(q^{\alpha+\gamma+n+1} ; q\right)_{n-k} q^{\left(\begin{array}{c}
k \\
2
\end{array}\right)}(-x)^{n-k}}{(q ; q)_{k}\left(q, q^{\alpha+1} ; q\right)_{n-k}}
$$

satisfy the orthogonality relation [23]

$$
\begin{aligned}
\sum_{k=0}^{\infty} \frac{\left(q^{k+1} ; q\right)_{\infty} q^{k(\alpha+1)}}{\left(q^{\gamma+k+1} ; q\right)_{\infty}} p_{m}\left(q^{k} ; q^{\alpha}, q^{\gamma} \mid q\right) p_{n}\left(q^{k} ; q^{\alpha}, q^{\gamma} \mid q\right) \\
\quad=\frac{\left(q, q^{\alpha+\gamma+n+1} ; q\right)_{\infty}(q ; q)_{n} q^{n(\alpha+1)} \delta_{m, n}}{\left(q^{\alpha+1}, q^{\gamma+n+1} ; q\right)_{\infty}\left(q^{\alpha+1} ; q\right)_{n}\left(1-q^{\alpha+\gamma+2 n+1}\right)} .
\end{aligned}
$$

This leads to the choice

$$
\begin{aligned}
& d \mu(x ; \gamma \mid q)=\sum_{k=0}^{\infty} \frac{(q x ; q)_{\infty} x}{\left(q^{\gamma+1} x ; q\right)_{\infty}} \delta\left(x-q^{k}\right) \\
& \zeta_{n}(\alpha, \gamma \mid q)=\frac{\left(q, q^{\alpha+\gamma+n+1} ; q\right)_{\infty}(q ; q)_{n} q^{n(\alpha+1)}}{\left(q^{\alpha+1}, q^{\gamma+n+1} ; q\right)_{\infty}\left(q^{\alpha+1} ; q\right)_{n}\left(1-q^{\alpha+\gamma+2 n+1}\right)} .
\end{aligned}
$$

Following our general construction we define the polynomials

$$
M_{m, n}^{(\beta, \gamma)}\left(z_{1}, z_{2} \mid q\right)= \begin{cases}z_{1}^{m-n} p_{n}\left(z_{1} z_{2}, q^{\beta+m-n}, q^{\gamma} \mid q\right), & m \geq n, \\ M_{n, m}^{(\beta, \gamma)}\left(z_{2}, z_{1} \mid q\right), & m<n .\end{cases}
$$


Then we have the explicit form

$$
\begin{aligned}
& M_{m, n}^{(\beta, \gamma)}\left(z_{1}, z_{2} \mid q\right)=\frac{(q ; q)_{n}\left(q^{\beta+1} ; q\right)_{m-n}}{\left(q^{\beta+\gamma+1} ; q\right)_{m} q^{\left(\begin{array}{c}
n \\
2
\end{array}\right)}} \sum_{k=0}^{n} \frac{\left(q^{\beta+\gamma+1} ; q\right)_{m+n-k} q^{\left(\begin{array}{c}
k \\
2
\end{array}\right)} z_{1}^{m-k}\left(-z_{2}\right)^{n-k}}{(q ; q)_{k}(q ; q)_{n-k}\left(q^{\beta+1} ; q\right)_{m-k}}, \\
& z_{1} M_{m, n}^{(\beta+1, \gamma)}\left(z_{1}, z_{2} \mid q\right)=M_{m+1, n}^{(\beta, \gamma)}\left(z_{1}, z_{2} \mid q\right)
\end{aligned}
$$

for $m \geq n$. Applying Theorem 2.1 we obtain:

Theorem 6.1. For $m, n, s, t=0,1, \ldots$ and $\beta, \gamma>-1$ we have the following orthogonality relation

$$
\int_{\mathbb{R}^{2}} M_{m, n}^{(\beta, \gamma)}(z, \bar{z} \mid q) \overline{M_{s, t}^{(\beta, \gamma)}(z, \bar{z} \mid q)} r^{2 \beta} d \mu\left(r^{2} ; \gamma \mid q\right) d \theta=\zeta_{m \wedge n}(|m-n|+\beta, \gamma \mid q) \delta_{m, s} \delta_{n, t},
$$

where $d \mu(x ; \gamma \mid q), \zeta_{n}(\alpha, \gamma \mid q)$ are given in (6.1).

Next we apply formulas (2.8)-(2.11) and (2.13) to

$$
c_{k}(n, \alpha)=\frac{(q ; q)_{n}\left(q^{\alpha+\gamma+n+1} ; q\right)_{n-k}(-1)^{n-k}}{(q ; q)_{k}\left(q, q^{\alpha+1} ; q\right)_{n-k} q^{\left(\begin{array}{c}
n \\
2
\end{array}\right)-\left(\begin{array}{c}
k \\
2
\end{array}\right)}},
$$

and obtain the following recurrences:

Theorem 6.2. For $\beta, \gamma>-1$ and $m \geq n$ we have

$$
\begin{aligned}
\frac{\left(1-q^{\beta+\gamma+m+n+2}\right)}{q^{n}\left(1-q^{\beta+m-n+1}\right)} z_{2} M_{m+1, n}^{(\beta, \gamma)}\left(z_{1}, z_{2} \mid q\right)=-M_{m+1, n+1}^{(\beta, \gamma)}\left(z_{1}, z_{2} \mid q\right)+M_{m, n}^{(\beta, \gamma)}\left(z_{1}, z_{2} \mid q\right), & \\
z_{1} M_{m, n}^{(\beta, \gamma)}\left(z_{1}, z_{2} \mid q\right)= & \frac{\left(1-q^{\beta+m+1}\right)\left(1-q^{\beta+\gamma+m+1}\right)}{\left(1-q^{\beta+m-n+1}\right)\left(1-q^{\beta+\gamma+m+n+1}\right)} M_{m+1, n}^{(\beta, \gamma)}\left(z_{1}, z_{2} \mid q\right) \\
& +\frac{q^{m-n+\beta+1}\left(1-q^{n}\right)\left(1-q^{\gamma+n}\right)}{\left(1-q^{\beta+m-n+1}\right)\left(1-q^{\beta+\gamma+m+n+1}\right)} M_{m, n-1}^{(\beta, \gamma)}\left(z_{1}, z_{2} \mid q\right) .
\end{aligned}
$$

The polynomials $\left\{p_{n}\left(x ; q^{\alpha}, q^{\gamma} \mid q\right)\right\}$ satisfy the following second-order difference equation

$$
\left(1+q^{\alpha}-\left(q^{1-n}+q^{n+\alpha+\gamma+2}\right) x\right) y(x q)=q^{\alpha}\left(1-q^{\gamma+2} x\right) y\left(q^{2} x\right)+(1-q x) y(x)
$$

or

$$
\begin{gathered}
\left(1-q^{\gamma+2} x\right) \theta_{q, x}^{2} y(x)+\frac{q^{-\alpha}-1-q^{1-n-\alpha} x-q^{\gamma+2}\left(2-q^{n}\right) x}{1-q} \theta_{q, x} y(x) \\
-\frac{1+q^{1-\alpha} x-\left(q^{1-n-\alpha}+q^{\gamma+n+2}\right) x}{(1-q)^{2}} y(x)=0,
\end{gathered}
$$

where $y(x)=p_{n}\left(x ; q^{\alpha}, q^{\gamma} \mid q\right), \theta_{q, z}=z D_{q, z}$.

Theorem 6.3. For $m \geq n$ and $\beta, \gamma>-1$, the function $f=M_{m, n}^{(\beta, \gamma)}\left(z_{1}, z_{2}\right)$ satisfies

$$
\begin{gathered}
\theta_{q, z_{2}}^{2} f+\left\{\frac{q^{n-m-\beta}-1-q^{1-m-\beta} z_{1} z_{2}-q^{\gamma+2}\left(2-q^{n}\right) z_{1} z_{2}}{(1-q)\left(1-q^{\gamma+2} z_{1} z_{2}\right)}\right\} \theta_{q, z_{2}} f \\
-\frac{1+\left(q^{n+1-m-\beta}-q^{1-m-\beta}-q^{\gamma+n+2}\right) z_{1} z_{2}}{(1-q)^{2}\left(1-q^{\gamma+2} z_{1} z_{2}\right)} f=0
\end{gathered}
$$

and

$$
\begin{aligned}
\theta_{q, z_{1}}^{2} f & +\frac{z_{1} z_{2}\left(2 q^{\gamma+2}-q^{1-n}-q^{m+\beta+\gamma+2}\right)+q^{m-n+\beta}-1}{(1-q)\left(1-q^{\gamma+2} z_{1} z_{2}\right)} \theta_{q, z_{1}} f \\
& +\frac{\left(q^{1-n}-q^{1+m-n+\beta}+q^{\gamma+2}\left(q^{m+\beta}+q^{2(m-n+\beta)}-1\right)\right) z_{1} z_{2}-q^{2(m-n+\beta)}}{(1-q)^{2}\left(1-q^{\gamma+2} z_{1} z_{2}\right)} f=0 .
\end{aligned}
$$


The polynomials $\left\{p_{n}\left(x ; q^{\alpha}, q^{\gamma} \mid q\right)\right\}$ satisfy the following difference equations

$$
\begin{aligned}
& p_{n}\left(x ; q^{\alpha}, q^{\gamma} \mid q\right)-p_{n}\left(q x ; q^{\alpha}, q^{\gamma} \mid q\right) \\
& \quad=-\frac{q^{1-n}\left(1-q^{n}\right)\left(1-q^{\alpha+\gamma+n-1}\right)}{1-q^{\alpha+1}} x p_{n-1}\left(x ; q^{\alpha+1}, q^{\gamma+1} \mid q\right), \\
& D_{q^{-1}, x}\left(w\left(x ; q^{\alpha}, q^{\gamma} \mid q\right) p_{n}\left(x ; q^{\alpha}, q^{\gamma} \mid q\right)=\frac{\left(1-q^{\alpha}\right) w\left(x ; q^{\alpha-1}, q^{\gamma-1} \mid q\right)}{q^{\alpha-1}(1-q)} p_{n+1}\left(x ; q^{\alpha-1}, q^{\gamma-1} \mid q\right),\right.
\end{aligned}
$$

where

$$
w\left(x ; q^{\alpha}, q^{\gamma} \mid q\right)=\frac{(q x ; q)_{\infty} x^{\alpha}}{\left(q^{\gamma+1} x ; q\right)_{\infty}} .
$$

Theorem 6.4. For $\beta, \gamma>-1$ we have

$$
M_{m, n}^{(\beta, \gamma)}\left(z_{1}, z_{2} \mid q\right)-M_{m, n}^{(\beta, \gamma)}\left(z_{1}, q z_{2} \mid q\right)=-\frac{q^{1-n}\left(1-q^{n}\right)\left(1-q^{m+\beta+\gamma-1}\right)}{1-q^{m-n+\beta}} z_{2} M_{m, n-1}^{(\beta, \gamma+1)}\left(z_{1}, z_{2} \mid q\right)
$$

for $m \geq n$, and

$$
\begin{aligned}
& D_{q^{-1}, z_{1}}\left(w\left(z_{1} z_{2} ; q^{\beta}, q^{\gamma} \mid q\right) M_{m, n}^{(\beta, \gamma)}\left(z_{1}, z_{2} \mid q\right)\right) \\
& =\frac{\left(1-q^{m-n+\beta}\right) w\left(z_{1} z_{2} ; q^{\beta}, q^{\gamma-1} \mid q\right)}{q^{m-n+\beta}(1-q)} M_{m, n+1}^{(\beta, \gamma-1)}\left(z_{1}, z_{2} \mid q\right), \\
& z_{2}^{n-m} D_{q^{-1}, z_{2}}\left(z_{2}^{m-n} w\left(z_{1} z_{2} ; q^{\beta}, q^{\gamma} \mid q\right) M_{m, n}^{(\beta, \gamma)}\left(z_{1}, z_{2} \mid q\right)\right) \\
& =\frac{z_{1}\left(1-q^{m-n+\beta}\right) w\left(z_{1} z_{2} ; q^{\beta}, q^{\gamma-1} \mid q\right)}{z_{2} q^{m-n+\beta-1}(1-q)} M_{m, n+1}^{(\beta, \gamma-1)}\left(z_{1}, z_{2} \mid q\right)
\end{aligned}
$$

for $m \geq n+1$.

The above recurrences are obtained by applying the definition of $M_{m, n}^{(\beta, \gamma)}\left(z_{1}, z_{2} \mid q\right)$ to $(6.2)$ and (6.3).

\section{Polynomial solutions to differential equations}

In this section we study polynomial solutions to partial differential equations. We are looking for polynomial solutions to the second-order partial differential equation (3.15). The results of this section should be contrasted with those in [9].

Let $f=\sum_{m, n=0}^{\infty} a_{j, k} z_{1}^{j} z_{2}^{k}$ and substitute in (3.15).

Theorem 7.1. The partial differential equation (3.15), namely,

$$
\partial_{\partial z_{1}} \partial_{\partial z_{2}} f+\left(\frac{\beta-z_{1} z_{2}}{z_{1}}\right) \partial_{\partial z_{2}} f=-n f
$$

has a power series solution

$$
f\left(z_{1}, z_{2}\right)=\sum_{j, k=0}^{\infty} a_{j, k} z_{1}^{j} z_{2}^{k}
$$

if and only if

$$
f\left(z_{1}, z_{2}\right)=\sum_{j=1}^{\infty} a_{j, 0} \frac{n ! z_{1}^{j}}{(\beta+j+1)_{n}} L_{n}^{(\beta+j)}\left(z_{1} z_{2}\right)+\sum_{k=0}^{n} a_{0, k} z_{22}^{k} F_{1}\left(\begin{array}{c}
-n+k, 1 \\
k+1, \beta+1
\end{array} \mid z_{1} z_{2}\right) .
$$


Proof. Substitute the power series (7.1) for $f$ in (3.15) and equate coefficients of like powers of $z_{1}$ and $z_{2}$ to find that

$$
a_{j, k}=\frac{k-1-n}{k(\beta+j)} a_{j-1, k-1}, \quad j, k>0 .
$$

We iterate this and find that

$$
a_{j, k}= \begin{cases}\frac{(-n)_{k} a_{j-k, 0}}{k !(\beta+j-k+1)_{k}}, & j \geq k, \\ \frac{(k-j-n)_{j}(k-j) !}{k !(\beta+1) j} a_{0, k-j}, & j \leq k .\end{cases}
$$

This proves the theorem.

Theorem 7.2. In order for the equation

$$
\partial_{z_{1}} \partial_{z_{2}} f+\left(\frac{\beta}{z_{1}}-z_{2}\right) \partial_{z_{2}} f=\lambda f
$$

to have a polynomials solution in $z_{2}$, it is necessary and sufficient that $\lambda=-n, n=0,1,2, \ldots$, in which case the function $f$ will be given as in Theorem 7.1.

\section{A biorthogonal system}

Theorem 8.1. Given three sets $A, B, C$ and a function $\rho$ from $C$ to $B$. For each $b \in B$, let $\left\{S, \mathcal{F}_{S}, d \mu(x)\right\}$ and $\left\{T, \mathcal{F}_{T}, d \nu(x)\right\}$ be two probability spaces such that $\left\{\varphi_{a}(x ; b) f(x, b)\right\}_{a \in A} \subset$ $L^{2}(S, d \mu(x))$ and $\left\{\psi_{c}(y)\right\}_{c \in C} \subset L^{2}(T, d \nu(y))$,

$$
\int_{S} \varphi_{a_{1}}(x ; b) \overline{\varphi_{a_{2}}(x ; b)}|f(x, b)|^{2} d \mu(x)=\zeta_{a_{1}}(b) \delta_{a_{1}, a_{2}}
$$

and

$$
\int_{T} \psi_{c_{1}}(y) \overline{\psi_{c_{2}}(y)} d \nu(y)=\eta_{c_{1}} \delta_{c_{1}, c_{2}}
$$

Then

$$
\int_{S \times T} \Phi_{a_{1}, c_{1}}(x, y) \overline{\Phi_{a_{2}, c_{2}}(x, y)} d \mu(x) \nu(y)=\zeta_{a_{1}}\left(\rho\left(c_{1}\right)\right) \eta_{c_{1}} \delta_{a_{1}, a_{2}} \delta_{c_{1}, c_{2}},
$$

where

$$
\Phi_{a_{1}, c_{1}}(x, y)=\varphi_{a_{1}}\left(x ; \rho\left(c_{1}\right)\right) \psi_{c_{1}}(y) f\left(x ; \rho\left(c_{1}\right)\right) .
$$

Proof. Observe that for each $(a, c) \in A \times C, \Phi_{a, c}(x, y) \in L^{2}(S \times T, d \mu(x) d \nu(y))$ by Fubini's theorem. Then for $a_{1}, a_{2} \in A, c_{1}, c_{2} \in C$ we have $\Phi_{a_{1}, c_{1}}(x, y) \overline{\Phi_{a_{2}, c_{2}}(x, y)} \in L(S \times T, d \mu(x) d \nu(y))$ by applying Cauchy-Schwartz inequality. By applying Fubini's theorem we get

$$
\begin{aligned}
& \int_{S \times T} \Phi_{a_{1}, c_{1}}(x, y) \overline{\Phi_{a_{2}, c_{2}}(x, y)} d \mu(x) \nu(y) \\
& =\int_{S} \varphi_{a_{1}}\left(x ; \rho\left(c_{1}\right)\right) f\left(x, \rho\left(c_{1}\right)\right) \overline{\varphi_{a_{2}}\left(x ; \rho\left(c_{2}\right)\right) f\left(x, \rho\left(c_{2}\right)\right)} d \mu(x) \int_{T} \psi_{c_{1}}(y) \overline{\psi_{c_{2}}(y)} d \nu(y) \\
& =\eta_{c_{1}} \delta_{c_{1}, c_{2}} \int_{S} \varphi_{a_{1}}\left(x ; \rho\left(c_{1}\right)\right) \overline{\varphi_{a_{2}}\left(x ; \rho\left(c_{1}\right)\right)}\left|f\left(x, \rho\left(c_{1}\right)\right)\right|^{2} d \mu(x)=\zeta_{a_{1}}\left(\rho\left(c_{1}\right)\right) \eta_{c_{1}} \delta_{a_{1}, a_{2}} \delta_{c_{1}, c_{2}} .
\end{aligned}
$$


For $a, b, c, d \in(-1,1)$, the Askey-Wilson polynomials

$$
p_{n}(\cos \theta ; a, b, c, d \mid q)={ }_{4} \phi_{3}\left(\begin{array}{c}
q^{-n}, a b c d q^{n-1}, a e^{i \theta}, a e^{-i \theta} \\
a b, a c, a d
\end{array} ; q, q\right),
$$

satisfy

$$
\begin{aligned}
\int_{-1}^{1} p_{m}(x ; a, b, c, d \mid q) p_{n}(x ; a, b, c, d \mid q) w(x ; a, b, c, d \mid q) d x \\
\quad=\frac{2 \pi\left(a b c d q^{2 n} ; q\right)_{\infty}\left(a b c d q^{n-1} ; q\right)_{n} \delta_{m, n}}{\left(q^{n+1} ; q\right)_{\infty}\left(a b q^{n}, a c q^{n}, a d q^{n}, b c q^{n}, b d q^{n}, c d q^{n} ; q\right)_{\infty}}
\end{aligned}
$$

where

$$
\begin{aligned}
& w(x ; a, b, c, d \mid q)=\frac{h(x, 1) h(x, \sqrt{q}) h(x,-1) h(x,-\sqrt{q})}{h(x, a) h(x, b) h(x, c) h(x, d) \sqrt{1-x^{2}}}, \\
& h(x, a)=\prod_{k=0}^{\infty}\left(1-2 a x q^{k}+a^{2} q^{2 k}\right), \quad x=\cos \theta .
\end{aligned}
$$

\section{Theorem 8.2. Let}

$$
\begin{aligned}
& u_{j, k}^{(\gamma, \delta)}\left(x, y ; a_{1}, b_{1}, c_{1}, d_{1} ; a_{2}, b_{2}, c_{2}, d_{2} \mid q\right)=p_{j}\left(x ; a_{1}, b_{1}, c_{1}, d_{1} q^{\gamma k+\delta} \mid q\right) p_{k}\left(y ; a_{2}, b_{2}, c_{2}, d_{2} \mid q\right), \\
& v_{j, k}^{(\gamma, \delta)}\left(x, y ; a_{1}, b_{1}, c_{1}, d_{1} ; a_{2}, b_{2}, c_{2}, d_{2} \mid q\right)=\frac{p_{j}\left(x ; a_{1}, b_{1}, c_{1}, d_{1} q^{\gamma k+\delta} \mid q\right) p_{k}\left(y ; a_{2}, b_{2}, c_{2}, d_{2} \mid q\right)}{h\left(x, d_{1} q^{\gamma k+\delta}\right)}, \\
& w\left(x, y ; a_{1}, b_{1}, c_{1} ; a_{2}, b_{2}, c_{2}, d_{2} \mid q\right) \\
& =\frac{h(x, 1) h(x, \sqrt{q}) h(x,-1) h(x,-\sqrt{q})}{h\left(x, a_{1}\right) h\left(x, b_{1}\right) h\left(x, c_{1}\right) \sqrt{1-x^{2}}} \frac{h(y, 1) h(y, \sqrt{q}) h(y,-1) h(y,-\sqrt{q})}{h\left(y, a_{2}\right) h\left(y, b_{2}\right) h\left(y, c_{2}\right) h\left(y, d_{2}\right) \sqrt{1-y^{2}}}, \\
& p_{j, k}^{(\alpha, \beta, \gamma, \delta)}\left(x, y ; a_{1}, b_{1}, c_{1}, d_{1} ; a_{2}, b_{2}, c_{2}, d_{2} \mid q\right) \\
& =\frac{p_{j}\left(x ; a_{1}, b_{1}, c_{1} q^{\alpha k+\beta}, d_{1} q^{\gamma k \delta} \mid q\right) p_{k}\left(y ; a_{2}, b_{2}, c_{2}, d_{2} \mid q\right)}{h\left(x, c_{1} q^{\alpha k+\beta}\right)}, \\
& q_{j, k}^{(\alpha, \beta, \gamma, \delta)}\left(x, y ; a_{1}, b_{1}, c_{1}, d_{1} ; a_{2}, b_{2}, c_{2}, d_{2} \mid q\right) \\
& =\frac{p_{j}\left(x ; a_{1}, b_{1}, c_{1} q^{\alpha k+\beta}, d_{1} q^{\gamma k \delta} \mid q\right) p_{k}\left(y ; a_{2}, b_{2}, c_{2}, d_{2} \mid q\right)}{h\left(x, d_{1} q^{\gamma k+\delta}\right)},
\end{aligned}
$$

and

$$
\begin{aligned}
& w\left(x, y ; a_{1}, b_{1} ; a_{2}, b_{2}, c_{2}, d_{2} \mid q\right) \\
& \quad=\frac{h(x, 1) h(x, \sqrt{q}) h(x,-1) h(x,-\sqrt{q})}{h\left(x, a_{1}\right) h\left(x, b_{1}\right) \sqrt{1-x^{2}}} \frac{h(y, 1) h(y, \sqrt{q}) h(y,-1) h(y,-\sqrt{q})}{h\left(y, a_{2}\right) h\left(y, b_{2}\right) h\left(y, c_{2}\right) h\left(y, d_{2}\right) \sqrt{1-y^{2}}},
\end{aligned}
$$

where

$$
x=\cos \theta, \quad y=\cos \varphi, \quad \alpha, \beta \geq 0,
$$

then

$$
\begin{aligned}
& \int_{-1}^{1} \int_{-1}^{1} u_{j, k}^{(\gamma, \delta)}\left(x, y ; a_{1}, b_{1}, c_{1}, d_{1} ; a_{2}, b_{2}, c_{2}, d_{2} \mid q\right) v_{m, n}^{(\gamma, \delta)}\left(x, y ; a_{1}, b_{1}, c_{1}, d_{1} ; a_{2}, b_{2}, c_{2}, d_{2} \mid q\right) \\
& \quad \times w\left(x, y ; a_{1}, b_{1}, c_{1} ; a_{2}, b_{2}, c_{2}, d_{2} \mid q\right) d x d y
\end{aligned}
$$




$$
\begin{aligned}
= & \frac{4 \pi^{2}\left(a_{2} b_{2} c_{2} d_{2} q^{2 k} ; q\right)_{\infty}\left(a_{2} b_{2} c_{2} d_{2} q^{k-1} ; q\right)_{k}}{\left(q^{k+1}, a_{2} b_{2} q^{k} ; q\right)_{\infty}\left(a_{2} c_{2} q^{k}, a_{2} d_{2} q^{k}, b_{2} c_{2} q^{k}, b_{2} d_{2} q^{k}, c_{2} d_{2} q^{k} ; q\right)_{\infty}} \\
& \times \frac{\left(a_{1} b_{1} c_{1} d_{1} q^{2 j+\gamma k+\delta} ; q\right)_{\infty}}{\left(q^{j+1}, a_{1} b_{1} q^{j}, a_{1} c_{1} q^{j+k}, a_{1} d_{1} q^{j+\gamma k+\delta} ; q\right)_{\infty}} \\
& \times \frac{\left(a_{1} b_{1} c_{1} d_{1} q^{j+\gamma k+\delta-1} ; q\right)_{j} \delta_{j, m} \delta_{k, n}}{\left(b_{1} c_{1} q^{j+k}, b_{1} d_{1} q^{j+\gamma k+\delta}, c_{1} d_{1} q^{j+\gamma k+\delta} ; q\right)_{\infty}} .
\end{aligned}
$$

and

$$
\begin{aligned}
\int_{-1}^{1} \int_{-1}^{1} & p_{j, k}^{(\alpha, \beta, \gamma, \delta)}\left(x, y ; a_{1}, b_{1}, c_{1}, d_{1} ; a_{2}, b_{2}, c_{2}, d_{2} \mid q\right) \\
& \times q_{m, n}^{(\alpha, \beta, \gamma, \delta)}\left(x, y ; a_{1}, b_{1}, c_{1}, d_{1} ; a_{2}, b_{2}, c_{2}, d_{2} \mid q\right) w\left(x, y ; a_{1}, b_{1} ; a_{2}, b_{2}, c_{2}, d_{2} \mid q\right) d x d y \\
= & \frac{4 \pi^{2}\left(a_{2} b_{2} c_{2} d_{2} q^{2 k} ; q\right)_{\infty}\left(a_{2} b_{2} c_{2} d_{2} q^{k-1} ; q\right)_{k}}{\left(q^{k+1}, a_{2} b_{2} q^{k} ; q\right)_{\infty}\left(a_{2} c_{2} q^{k}, a_{2} d_{2} q^{k}, b_{2} c_{2} q^{k}, b_{2} d_{2} q^{k}, c_{2} d_{2} q^{k} ; q\right)_{\infty}} \\
& \times \frac{\left(a_{1} b_{1} c_{1} d_{1} q^{2 j+(\alpha+\gamma) k+\beta+\delta} ; q\right)_{\infty}}{\left(q^{j+1}, a_{1} b_{1} q^{j}, a_{1} c_{1} q^{j+\alpha k+\beta}, a_{1} d_{1} q^{j+\gamma k+\delta} ; q\right)_{\infty}} \\
& \times \frac{\left(a_{1} b_{1} c_{1} d_{1} q^{j+(\alpha+\gamma) k+\beta+\delta-1} ; q\right)_{j} \delta_{j, m} \delta_{k, n}}{\left(b_{1} c_{1} q^{j+\alpha k+\beta}, b_{1} d_{1} q^{j+\gamma k+\delta}, c_{1} d_{1} q^{j+(\alpha+\gamma) k+\beta+\delta} ; q\right)_{\infty}}
\end{aligned}
$$

Proof. The details of the calculation are

$$
\begin{aligned}
\int_{-1}^{1} \int_{-1}^{1} & p_{j, k}^{(\alpha, \beta, \gamma, \delta)}\left(x, y ; a_{1}, b_{1}, c_{1}, d_{1} ; a_{2}, b_{2}, c_{2}, d_{2} \mid q\right) \\
& \times q_{m, n}^{(\alpha, \beta, \gamma, \delta)}\left(x, y ; a_{1}, b_{1}, c_{1}, d_{1} ; a_{2}, b_{2}, c_{2}, d_{2} \mid q\right) w\left(x, y ; a_{1}, b_{1} ; a_{2}, b_{2}, c_{2}, d_{2} \mid q\right) d x d y \\
= & \int_{-1}^{1} p_{j}\left(x ; a_{1}, b_{1}, c_{1} q^{\alpha k+\beta}, d_{1} q^{\gamma k+\delta} \mid q\right) p_{m}\left(x ; a_{1}, b_{1}, c_{1} q^{\alpha n+\beta}, d_{1} q^{\gamma n+\delta} \mid q\right) \\
& \times \frac{h(x, 1) h(x, \sqrt{q}) h(x,-1) h(x,-\sqrt{q}) d x}{h\left(x, a_{1}\right) h\left(x, b_{1}\right) h\left(x, c_{1} q^{\alpha k+\beta}\right) h\left(x, d_{1} q^{\gamma n+\delta}\right) \sqrt{1-x^{2}}} \\
& \times \int_{-1}^{1} p_{k}\left(y ; a_{2}, b_{2}, c_{2}, d_{2} \mid q\right) p_{n}\left(y ; a_{2}, b_{2}, c_{2}, d_{2} \mid q\right) w\left(y ; a_{2}, b_{2}, c_{2}, d_{2} \mid q\right) d y \\
= & \frac{2 \pi\left(a_{2} b_{2} c_{2} d_{2} q^{2 k} ; q\right)_{\infty}\left(a_{2} b_{2} c_{2} d_{2} q^{k-1} ; q\right)_{k} \delta_{k, n}}{\left(q^{k+1} ; q\right)_{\infty}\left(a_{2} b_{2} q^{k}, a_{2} c_{2} q^{k}, a_{2} d_{2} q^{k}, b_{2} c_{2} q^{k}, b_{2} d_{2} q^{k}, c_{2} d_{2} q^{k} ; q\right)_{\infty}} \\
& \times \int_{-1}^{1} p_{j}\left(x ; a_{1}, b_{1}, c_{1} q^{\alpha k+\beta}, d_{1} q^{\gamma k+\delta} \mid q\right) p_{m}\left(x ; a_{1}, b_{1}, c_{1} q^{\alpha k+\beta}, d_{1} q^{\gamma k+\delta} \mid q\right) \\
& \times \frac{h(x, 1) h(x, \sqrt{q}) h(x,-1) h(x,-\sqrt{q}) d x}{h\left(x, a_{1}\right) h\left(x, b_{1}\right) h\left(x, c_{1} q^{\alpha k+\beta}\right) h\left(x, d_{1} q^{\gamma k+\delta}\right) \sqrt{1-x^{2}}} \\
= & \frac{4 \pi^{2}\left(a_{2} b_{2} c_{2} d_{2} q^{2 k} ; q\right)_{\infty}\left(a_{2} b_{2} c_{2} d_{2} q^{k-1} ; q\right)_{k}}{\left(q^{k+1}, a_{2} b_{2} q^{k} ; q\right)_{\infty}\left(a_{2} c_{2} q^{k}, a_{2} d_{2} q^{k}, b_{2} c_{2} q^{k}, b_{2} d_{2} q^{k}, c_{2} d_{2} q^{k} ; q\right)_{\infty}} \\
& \times \frac{\left(a_{1} b_{1} c_{1} d_{1} q^{2 j+(\alpha+\gamma) k+\beta+\delta} ; q\right)_{\infty}}{\left(q^{j+1}, a_{1} b_{1} q^{j}, a_{1} c_{1} q^{j+\alpha k+\beta}, a_{1} d_{1} q^{j+\gamma k+\delta} ; q\right)_{\infty}} \\
& \times \frac{\left(a_{1} b_{1} c_{1} d_{1} q^{j+(\alpha+\gamma) k+\beta+\delta-1} ; q\right)_{j} \delta_{j, m} \delta_{k, n}}{\left(b_{1} c_{1} q^{j+\alpha k+\beta}, b_{1} d_{1} q^{j+\gamma k+\delta}, c_{1} d_{1} q^{j+(\alpha+\gamma) k+\beta+\delta} ; q\right)_{\infty}} .
\end{aligned}
$$

The $u, v$ orthogonality can be proved similarly.

\section{Theorem 8.3. Let}

$$
p_{j, k}^{(\gamma, \delta)}\left(x, y ; a_{1}, b_{1}, c_{1}, d_{1} ; a_{2}, b_{2}, c_{2}, d_{2} \mid q\right)=\frac{p_{j}\left(x ; a_{1}, b_{1}, c_{1}, d_{1} q^{\gamma k+\delta} \mid q\right) p_{k}\left(y ; a_{2}, b_{2}, c_{2}, d_{2} \mid q\right)}{\left(d_{1} q^{\gamma k+\delta} e^{i \theta} ; q\right)_{\infty}} .
$$


Then

$$
\begin{aligned}
\int_{-1}^{1} \int_{-1}^{1} & p_{j, k}^{(\gamma, \delta)}\left(x, y ; a_{1}, b_{1}, c_{1}, d_{1} ; a_{2}, b_{2}, c_{2}, d_{2} \mid q\right) \\
& \times \frac{p_{m, n}^{(\gamma, \delta)}\left(x, y ; a_{1}, b_{1}, c_{1}, d_{1} ; a_{2}, b_{2}, c_{2}, d_{2} \mid q\right)}{w} w\left(x, y ; a_{1}, b_{1}, c_{1} ; a_{2}, b_{2}, c_{2}, d_{2} \mid q\right) d x d y \\
= & \frac{4 \pi^{2} \delta_{j, m} \delta_{k, n}}{\left(q^{m+1}, a_{1} b_{1} q^{m}, a_{1} c_{1} q^{m}, b_{1} c_{1} q^{m}, b_{1} c_{1} q^{m} ; q\right)_{\infty}} \\
& \times \frac{\left(a_{1} b_{1} c_{1} d_{1} q^{2 m+\gamma n+\delta} ; q\right)_{\infty}\left(a_{1} b_{1} c_{1} d_{1} q^{m+\gamma n+\delta-1} ; q\right)_{m}}{\left(a_{1} d_{1} q^{m+\gamma n+\delta}, b_{1} d_{1} q^{m+\gamma n+\delta}, c_{1} d_{1} q^{m+\gamma n+\delta} ; q\right)_{\infty}} \\
& \times \frac{\left(a_{2} b_{2} c_{2} d_{2} q^{2 n} ; q\right)_{\infty}\left(a_{2} b_{2} c_{2} d_{2} q^{n-1} ; q\right)_{n}}{\left(q^{n+1}, a_{2} b_{2} q^{n}, a_{2} c_{2} q^{n}, a_{2} d_{2} q^{n}, b_{2} c_{2} q^{n}, b_{2} d_{2} q^{n}, c_{2} d_{2} q^{n} ; q\right)_{\infty}} .
\end{aligned}
$$

where $x=\cos \theta, y=\cos \varphi, \alpha, \beta \geq 0$ and $w\left(x, y ; a_{1}, b_{1}, c_{1} ; a_{2}, b_{2}, c_{2}, d_{2} \mid q\right)$ is the same as in Theorem 8.2.

Proof. Observe that the integral

$$
\begin{aligned}
& \int_{-1}^{1} \int_{-1}^{1} \frac{p_{j, k}^{(\gamma, \delta)}\left(x, y ; a_{1}, b_{1}, c_{1}, d_{1} ; a_{2}, b_{2}, c_{2}, d_{2} \mid q\right)}{}=\left(x, y ; a_{1}, b_{1}, c_{1} ; a_{2}, b_{2}, c_{2}, d_{2} \mid q\right. \\
& \left.\quad \times \frac{p_{m, n}^{(\gamma, \delta)}\left(x, y ; a_{1}, b_{1}, c_{1}, d_{1} ; a_{2}, b_{2}, c_{2}, d_{2} \mid q\right)}{2}\right) d x d y
\end{aligned}
$$

equals

$$
\begin{aligned}
\int_{-1}^{1} p_{j}( & \left.x ; a_{1}, b_{1}, c_{1}, d_{1} q^{\gamma k+\delta} \mid q\right) p_{m}\left(x ; a_{1}, b_{1}, c_{1}, d_{1} q^{\gamma n+\delta} \mid q\right) \\
& \times \frac{h(x, 1) h(x, \sqrt{q}) h(x,-1) h(x,-\sqrt{q}) d x}{h\left(x, a_{1}\right) h\left(x, b_{1}\right) h\left(x, c_{1}\right)\left(d_{1} q^{\gamma k+\delta} e^{i \theta}, d_{1} q^{\gamma n+\delta} e^{-i \theta} ; q\right)_{\infty} \sqrt{1-x^{2}}} \\
& \times \int_{-1}^{1} p_{k}\left(y ; a_{2}, b_{2}, c_{2}, d_{2} \mid q\right) p_{n}\left(y ; a_{2}, b_{2}, c_{2}, d_{2} \mid q\right) w\left(y ; a_{2}, b_{2}, c_{2}, d_{2} \mid q\right) d y \\
= & \frac{2 \pi \delta_{k, n}\left(a_{2} b_{2} c_{2} d_{2} q^{2 n} ; q\right)_{\infty}\left(a_{2} b_{2} c_{2} d_{2} q^{n-1} ; q\right)_{n}}{\left(q^{n+1} ; q\right)_{\infty}\left(a_{2} b_{2} q^{n}, a_{2} c_{2} q^{n}, a_{2} d_{2} q^{n}, b_{2} c_{2} q^{n}, b_{2} d_{2} q^{n}, c_{2} d_{2} q^{n} ; q\right)_{\infty}} \\
& \times \int_{-1}^{1} p_{j}\left(x ; a_{1}, b_{1}, c_{1}, d_{1} q^{\gamma n+\delta} \mid q\right) p_{m}\left(x ; a_{1}, b_{1}, c_{1}, d_{1} q^{\gamma n+\delta} \mid q\right) \\
& \times \frac{h(x, 1) h(x, \sqrt{q}) h(x,-1) h(x,-\sqrt{q}) d x}{h\left(x, a_{1}\right) h\left(x, b_{1}\right) h\left(x, c_{1}\right) h\left(x, d_{1} q^{\gamma n+\delta}\right) \sqrt{1-x^{2}}} \\
= & \frac{4 \pi^{2} \delta_{j, m} \delta_{k, n}}{\left(q^{m+1}, a_{1} b_{1} q^{m}, a_{1} c_{1} q^{m}, b_{1} c_{1} q^{m}, b_{1} c_{1} q^{m} ; q\right)_{\infty}} \\
& \times \frac{\left(a_{1} b_{1} c_{1} d_{1} q^{2 m+\gamma n+\delta} ; q\right)_{\infty}\left(a_{1} b_{1} c_{1} d_{1} q^{m+\gamma n+\delta-1} ; q\right)_{m}}{\left(a_{1} d_{1} q^{m+\gamma n+\delta}, b_{1} d_{1} q^{m+\gamma n+\delta}, c_{1} d_{1} q^{m+\gamma n+\delta} ; q\right)_{\infty}} \\
& \times \frac{\left(a_{2} b_{2} c_{2} d_{2} q^{2 n} ; q\right)_{\infty}\left(a_{2} b_{2} c_{2} d_{2} q^{n-1} ; q\right)_{n}}{\left(q^{n+1}, a_{2} b_{2} q^{n}, a_{2} c_{2} q^{n}, a_{2} d_{2} q^{n}, b_{2} c_{2} q^{n}, b_{2} d_{2} q^{n}, c_{2} d_{2} q^{n} ; q\right)_{\infty}}
\end{aligned}
$$

\section{Acknowledgements}

The authors are very grateful to the anonymous referees for their detailed reports on the first draft of this paper. Research of M.E.H. Ismail supported by a grant from DSFP program at King Saud and by the National Plan for Science, Technology and innovation (MAARIFAH), King Abdelaziz City for Science and Technology, Kingdom of Saudi Arabia, Award number 14-MAT623-02. Research of R. Zhang partially supported by National Science Foundation of China, grant No. 11371294. 


\section{References}

[1] Al-Salam W.A., Chihara T.S., Convolutions of orthonormal polynomials, SIAM J. Math. Anal. 7 (1976), $16-28$.

[2] Ali S.T., Bagarello F., Honnouvo G., Modular structures on trace class operators and applications to Landau levels, J. Phys. A: Math. Theor. 43 (2010), 105202, 17 pages, arXiv:0906.3980.

[3] Appell P., Sur des polynômes de deux variables analogues aux polynômes de Jacobi, Archiv Math. Phys. 66 (1881), 238-245.

[4] Appell P., Kampé de Fériet J., Fonctions hypergéométriques et hypersphériques. Polynomes d'Hermite, Gauthier-Villars, Paris, 1926.

[5] Chihara T.S., An introduction to orthogonal polynomials, Mathematics and its Applications, Vol. 13, Gordon and Breach Science Publishers, New York - London - Paris, 1978.

[6] Cotfas N., Gazeau J.P., Górska K., Complex and real Hermite polynomials and related quantizations, J. Phys. A: Math. Theor. 43 (2010), 305304, 14 pages, arXiv:1001.3248.

[7] Dunkl C.F., Xu Y., Orthogonal polynomials of several variables, 2nd ed., Encyclopedia of Mathematics and its Applications, Cambridge University Press, Cambridge, 2014.

[8] Erdélyi A., Magnus W., Oberhettinger F., Tricomi F.G., Higher transcendental functions. Vol. II, McGrawHill Book Company, New York, 1953.

[9] Finkel F., Kamran N., The Lie algebraic structure of differential operators admitting invariant spaces of polynomials, Adv. in Appl. Math. 20 (1998), 300-322, q-alg/9612027.

[10] Floris P.G.A., A noncommutative discrete hypergroup associated with $q$-disk polynomials, J. Comput. Appl. Math. 68 (1996), 69-78.

[11] Floris P.G.A., Koelink H.T., A commuting $q$-analogue of the addition formula for disk polynomials, Constr. Approx. 13 (1997), 511-535, math.QA/9509222.

[12] Ghanmi A., A class of generalized complex Hermite polynomials, J. Math. Anal. Appl. 340 (2008), 13951406, arXiv:0704.3576.

[13] Ghanmi A., Operational formulae for the complex Hermite polynomials $H_{p, q}(z, \bar{z})$, Integral Transforms Spec. Funct. 24 (2013), 884-895, arXiv:1211.5746.

[14] Hermite C., Sur un nouveau développement en series des fonctions, Comptes Rendus Acad. Sci. Paris 58 (1864), 93-100, 266-273.

[15] Hermite C., Sur quelques développement en series des fonctions,, Comptes Rendus Acad. Sci. Paris 60 (1865), 370-377, 432-440, 461-466, 512-518.

[16] Intissar A., Intissar A., Spectral properties of the Cauchy transform on $L_{2}\left(\mathbb{C}, e^{-|z|^{2}} \lambda(z)\right)$, J. Math. Anal. Appl. 313 (2006), 400-418.

[17] Ismail M.E.H., Classical and quantum orthogonal polynomials in one variable, Encyclopedia of Mathematics and its Applications, Vol. 98, Cambridge University Press, Cambridge, 2009.

[18] Ismail M.E.H., Analytic properties of complex Hermite polynomials, Trans. Amer. Math. Soc. 368 (2016), 1189-1210.

[19] Ismail M.E.H., Zeng J., A combinatorial approach to the 2D-Hermite and 2D-Laguerre polynomials, Adv. in Appl. Math. 64 (2015), 70-88.

[20] Ismail M.E.H., Zeng J., Two variable extensions of the Laguerre and disc polynomials, J. Math. Anal. Appl. 424 (2015), 289-303.

[21] Ismail M.E.H., Zeng J., On some $2 D$ orthogonal q-polynomials, Trans. Amer. Math. Soc., to appear, arXiv:1411.5223.

[22] Itô K., Complex multiple Wiener integral, Japan J. Math. 22 (1952), 63-86.

[23] Koekoek R., Swarttouw R.F., The Askey-scheme of hypergeometric orthogonal polynomials and its $q$ analogue, Report 98-17, Faculty of Technical Mathematics and Informatics, Delft University of Technology, 1998, available at http://aw.twi.tudelft.nl/ koekoek/askey/.

[24] Koornwinder T.H., The addition formula for Jacobi polynomials III. Completion of the proof, Report TW 135/72, Mathematisch Centrum, Amsterdam, 1972, available at https://staff.fnwi.uva.nl/t.h. koornwinder/art/1972/addition3.pdf. 
[25] Koornwinder T.H., Two-variable analogues of the classical orthogonal polynomials, in Theory and Application of Special Functions (Proc. Advanced Sem., Math. Res. Center, Univ. Wisconsin, Madison, Wis., 1975), Academic Press, New York, 1975, 435-495.

[26] Maldonado C.D., Note on orthogonal polynomials which are "invariant in form" to rotations of axes, J. Math. Phys. 6 (1965), 1935-1938.

[27] Myrick D.R., A generalization of the radial polynomials of F. Zernike, SIAM J. Appl. Math. 14 (1966), 476-489.

[28] Rainville E.D., Special functions, The Macmillan Co., New York, 1960.

[29] Šapiro R.L., Special functions related to representations of the group $\mathrm{SU}(n)$, of class I with respect to $\mathrm{SU}(n-1)(n \geq 3)$, Izv. Vyssh. Uchebn. Zaved. Matematika (1968), no. 4, 97-107.

[30] Shigekawa I., Eigenvalue problems for the Schrödinger operator with the magnetic field on a compact Riemannian manifold, J. Funct. Anal. 75 (1987), 92-127.

[31] Szegö G., Orthogonal polynomials, Colloquium Publications, Vol. 23, 4th ed., Amer. Math. Soc., Providence, R.I., 1975.

[32] Thirulogasanthar K., Honnouvo G., Krzyżak A., Coherent states and Hermite polynomials on quaternionic Hilbert spaces, J. Phys. A: Math. Theor. 43 (2010), 385205, 13 pages.

[33] Waldron S., Orthogonal polynomials on the disc, J. Approx. Theory 150 (2008), 117-131.

[34] Wünsche A., Laguerre 2D-functions and their application in quantum optics, J. Phys. A: Math. Gen. 31 (1998), 8267-8287.

[35] Wünsche A., Transformations of Laguerre 2D-polynomials and their applications to quasiprobabilities, J. Phys. A: Math. Gen. 32 (1999), 3179-3199.

[36] Wünsche A., Generalized Zernike or disc polynomials, J. Comput. Appl. Math. 174 (2005), $135-163$.

[37] Xu Y., Complex versus real orthogonal polynomials of two variables, Integral Transforms Spec. Funct. 26 (2015), 134-151, arXiv:1307.7819.

[38] Zernike F., Beugungstheorie des Schneidenverfahrens und seiner verbesserten Form, der Phasenkontrastmethode, Physica 1 (1934), 689-704.

[39] Zernike F., Brinkman H.C., Hypersphärische Funktionen und die in sphärischen Bereichen orthogonalen Polynome, Proc. Akad. Wet. Amsterdam 38 (1935), 161-170. 